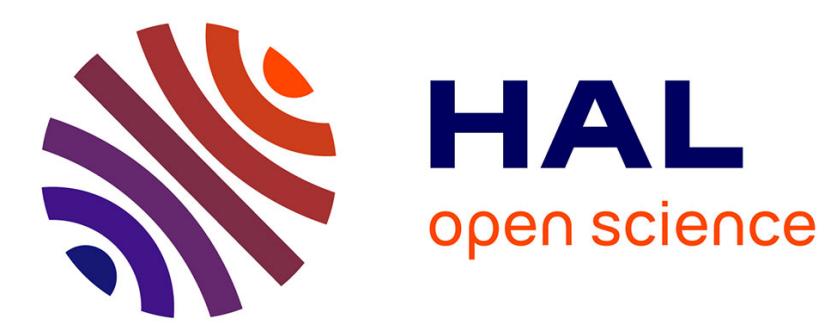

\title{
Évolution de la taille des familles au fil des générations en France (1850-1966)
}

Sandra Brée

\section{To cite this version:}

Sandra Brée. Évolution de la taille des familles au fil des générations en France (1850-1966). Population (édition française), 2017, 72 (2), 10.3917/popu.1702.0309 . halshs-01624678

\section{HAL Id: halshs-01624678 \\ https://shs.hal.science/halshs-01624678}

Submitted on 30 Jun 2021

HAL is a multi-disciplinary open access archive for the deposit and dissemination of scientific research documents, whether they are published or not. The documents may come from teaching and research institutions in France or abroad, or from public or private research centers.
L'archive ouverte pluridisciplinaire $\mathbf{H A L}$, est destinée au dépôt et à la diffusion de documents scientifiques de niveau recherche, publiés ou non, émanant des établissements d'enseignement et de recherche français ou étrangers, des laboratoires publics ou privés. 


\title{
Évolution de la taille des familles au fil des générations en France (1850-1966)
}

\begin{abstract}
Pour retracer la fécondité sur une longue période, les démographes et historiens ont souvent recours à des indicateurs synthétiques tels que la descendance finale ou l'indice conjoncturel de fécondité. Ces indicateurs décrivent un nombre moyen d'enfants par femme pour une génération ou à un moment donné, mais ne permettent pas de rendre compte de l'évolution des tailles des familles. La proportion des femmes sans enfant a-t-elle changé? Que représentent les familles de quatre enfants? Mobilisant huit recensements et enquêtes Familles dont elle compare minutieusement les questions et les données, Sandra BRÉE décrit les évolutions de ces différents types de familles dont les femmes sont nées entre les années 1850 et les années 1960, couvrant plus d'un siècle de générations. Elle montre la diminution des familles nombreuses dès la fin $d u$ XIX siècle, la non-uniformité de l'infécondité sur la période ainsi que l'augmentation des familles de deux enfants dès les générations nées dans les années 1920. Cette étude montre l'intérêt d'enrichir les indicateurs de fécondité moyens par des indicateurs de parité.
\end{abstract}

L'histoire de la fécondité française depuis le XIX ${ }^{e}$ siècle est aujourd'hui bien connue. Pourtant, les recherches qui lui sont consacrées font rarement référence à la distribution du nombre d'enfants qu'ont eus les femmes au cours de leur vie, autrement dit à la taille des familles ou parité. La fécondité est davantage abordée à travers le nombre moyen d'enfants par femme (descendance finale dans les générations, indice conjoncturel de fécondité pour l'analyse par période) et les taux de fécondité par âge, mais plus rarement en décomposant cette moyenne pour décrire combien de femmes n'ont eu aucun enfant, un enfant, deux enfants, etc. Lanalyse par moyenne cache pourtant l'évolution particulière de chaque type de famille. La baisse de la descendance finale est-elle due à une raréfaction des grandes familles ou à une plus grande fréquence des petites familles? Et qu'en est-il de l'infécondité? Quel est son poids dans l'évolution de la descendance finale?

\footnotetext{
* Centre de recherche en démographie, Université catholique de Louvain, Belgique.

Correspondance : Sandra Brée, Centre de recherche en démographie, Université catholique de Louvain, 1 place Montesquieu, B1348 Louvain-la-Neuve, Belgique, courriel : sandra.bree@uclouvain.be
} 
Les auteurs ayant analysé la fécondité des générations françaises sur le long terme (Daguet, 2002; Festy, 1979; Ined, 1991; Sardon, 1990; Toulemon, 2001) ont essentiellement travaillé à partir des données agrégées d'état civil (effectifs des naissances au numérateur) et des résultats des recensements (nombre de femmes au dénominateur). Ces données agrégées permettent de décrire l'évolution de la fécondité, mais pas de connaître le nombre précis d'enfants à la fin de la vie féconde des femmes ${ }^{(1)}$. Pourtant, comme le souligne Patrick Festy (1979, p. 96), « c'est le changement de la composition des familles qui est au cour de l'explication ». Seul Laurent Toulemon (1995, 2001) a utilisé les enquêtes Famille pour estimer la fécondité à partir des générations nées vers 1930 et calculé la composition des familles. Par ailleurs, ces analyses ne distinguent presque jamais les femmes mariées de celles restées célibataires.

Il n'existe donc pas de recherche sur la fécondité comparée des générations qui à la fois utilise une méthode rétrospective, remonte aux générations 1850 et distingue la fécondité des femmes mariées (ou l'ayant été : veuves ou

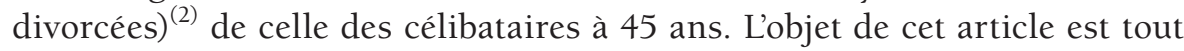
d'abord de faire le point sur les sources et données disponibles et les méthodes utilisées pour l'analyse de la fécondité des générations nées entre 1850 et 1965 en France. L'analyse porte ensuite sur l'évolution de la fécondité des générations sur une longue période à partir de la taille des familles, puis des probabilités d'agrandissement. L'étude de l'évolution conjointe de la taille des familles et de la descendance finale améliore la compréhension des tendances de la fécondité, et plus particulièrement le poids de l'évolution de chaque taille de famille dans l'évolution générale de la fécondité.

\section{Sources et méthode pour mesurer la taille des familles}

Le principe de la méthode d'estimation rétrospective de la fécondité consiste à utiliser les données collectées sur le nombre d'enfants que les femmes âgées d'au moins 45 ans déclarent avoir eu au cours de leur vie féconde. Cette question n'est pas posée dans les recensements de tous les pays ni à toutes les époques (Eggerickx et Begeot, 1993). La France offre un cas particulier. En effet, la question « Combien d'enfants avez-vous eus?» est posée lors des

\footnotetext{
(1) La vaste analyse de Patrick Festy (1979) sur la fécondité des pays occidentaux de 1870 à 1970 s'appuie sur une lecture en diagonale des taux quinquennaux de fécondité par âge collectés par Pierre Depoid (1941) pour évaluer la fécondité des générations françaises, méthode mobilisée également par Fabienne Daguet (2002) à partir des données du « rapport sur la situation démographique de la France en $1974 »$ (Ined, 1976).

(2) La vie féconde est généralement mesurée entre les âges de 15 et 50 ans. Nous avons choisi de la limiter à 45 ans (également souvent utilisée) car cela permet d'inclure une génération quinquennale de plus à l'analyse en ne changeant quasiment pas les résultats (puisque le nombre d'enfants nés de mère âgée de 45 à 50 ans est très faible).
} 
recensements de 1906, 1931 et $1946^{(3)}$. En 1931 et 1946, la taille des familles est fournie pour les femmes mariées, veuves et divorcées et les hommes veufs (pour les chefs de famille en 1906). Ce sont ces informations qui sont intéressantes pour l'analyse de la fécondité. Elles seront, à partir de 1954, transférées dans des enquêtes spécifiques dites enquêtes Famille, comme le préconisait Louis Henry (1953). Ce dernier proposait que l'étude approfondie de la fécondité se fasse à partir de « toute l'histoire de la famille [recueillie dans] des enquêtes spéciales » (Henry, 1953, p. 489-490) et de garder la question « combien d'enfants avez-vous? » dans les recensements quinquennaux pour connaître les statistiques des familles bénéficiaires des allocations familiales suivant le nombre d'enfants à charge.

\section{Quelles sont les données disponibles?}

À partir de 1954, la question du nombre d'enfants mis au monde n'est donc plus posée dans les recensements mais dans les enquêtes Famille qui leurs sont associées (tableau 1) et qui ne concernent qu'un échantillon de la population $^{(4)}$. La première a été organisée conjointement au recensement de 1954, puis se sont succédées celles de 1962, 1975, 1982, 1990, 1999 et 2011 (tableau 1). Les trois premières enquêtes n'ont concerné que les femmes déjà mariées ${ }^{(5)}$. Les suivantes concernent toutes les femmes, quel que soit leur état matrimonial, et les deux dernières les hommes également.

Tableau 1. Description des enquêtes Famille

\begin{tabular}{|c|c|c|c|c|}
\hline \multirow{2}{*}{$\begin{array}{l}\text { Date de } \\
\text { l'enquête }\end{array}$} & \multirow{2}{*}{ Générations } & \multirow{2}{*}{ Champ } & \multicolumn{2}{|c|}{ Effectif } \\
\hline & & & Femmes & Hommes \\
\hline 1954 & 1899-1908 & $\begin{array}{l}\text { Femmes mariées, } \\
\text { veuves, divorcées }\end{array}$ & 52474 & non enquêtés \\
\hline 1962 & \multicolumn{4}{|c|}{ Non diffusée } \\
\hline 1975 & 1910-1960 & $\begin{array}{l}\text { Femmes mariées, } \\
\text { veuves, divorcées }\end{array}$ & 247000 & non enquêtés \\
\hline 1982 & 1917-1963 & $\begin{array}{l}\text { Femmes } \\
\text { de } 18 \text { à } 64 \text { ans }\end{array}$ & 310000 & non enquêtés \\
\hline 1990 & 1921-1971 & $\begin{array}{c}\text { Femmes } \\
\text { de } 18 \text { à } 64 \text { ans }\end{array}$ & 340000 & non enquêtés \\
\hline 1999 & 1981 & Tous & 278000 & 167000 \\
\hline 2011 & 1993 & Tous & 238000 & 121000 \\
\hline
\end{tabular}

(3) La question « combien d'enfants avez-vous? » est posée dans les recensements à partir de 1886 mais elle ne fournit des informations que sur les enfants survivants et non les enfants nés vivants, ce qui peut constituer un biais important (pour Paris : Brée, 2011, 2017). Pour un historique de la statistique des familles, voir le chapitre premier de la statistique des familles du recensement de la population de 1946 (Insee, 1946, p. VIII-XII).

(4) La taille des échantillons par génération selon les différentes enquêtes est présentée dans la figure annexe A.1.

(5) L'enquête de 1962 n'est plus diffusée par l'Insee qui la considère biaisée. 
L'estimation de la fécondité à partir de questions rétrospectives peut être affectée par une série de biais liés à des erreurs de mémoire (en particulier pour les femmes les plus âgées au moment du recensement); à des effets de sélection induits par la mortalité des enfants et de leur mère, ainsi que la migration des mères; aux non-réponses. Les analyses menées pour la France et la Belgique montrent que les risques de biais liés aux erreurs de mémoire et aux effets de sélection sont très limités (Brée et al., 2016b), ce qui confirme les conclusions de Neels (2010) et Van Bavel (2014) pour la Belgique et celles d'Andersson et Sobolev (2013) pour la Suède. Les descendances finales des femmes calculées à partir des recensements et des enquêtes s'ajustent bien aux descendances finales issues des données de l'état civil. De plus, la comparaison de la fécondité des femmes d'une même génération observée à différents recensements ou enquêtes montre une bonne adéquation. Il apparaît cependant que les données issues des enquêtes sont moins fiables que celles des recensements exhaustifs, notamment pour les analyses à l'échelle régionale pour lesquelles les effectifs peuvent être assez réduits (Brée et al., $2016 b)^{(6)}$.

Quant aux biais concernant les non-réponses, ils avaient déjà posé question à Paul Vincent (1946). Il distingue deux types de non-répondantes à la question « combien avez-vous eu d'enfants nés vivants? » : les négligentes (ayant simplement oublié de répondre à la question, ou n'ayant pas voulu, mais cette option n'est pas vraiment envisagée) et celles ayant cru répondre « zéro » en n'inscrivant aucune mention. Après une analyse des non-réponses, il considère que les personnes vraiment négligentes ne représentent que $3 \%$ des non-réponses (en 1931). Il considère les 97 \% restantes comme sans enfant et ventile les $3 \%$ de non-réponses proportionnellement à la répartition des autres types de familles. Louis Henry remet en question cette méthode de ventilation (Henry, 1953). Il admet cependant que, si la solution de considérer tous les «non-déclarés » comme sans enfant n'est pas satisfaisante, il n'est pourtant pas possible de faire mieux. Les chercheurs en charge de la statistique des familles issue du recensement de 1946 ont également considéré que toutes les femmes n'ayant pas répondu à la question « combien avez-vous eu d'enfants nés vivants? » n'ont pas eu d'enfant. Si cette hypothèse peut sembler forte, les écarts entre les résultats du recensement de 1931 et celui de 1946 sont très réduits (figure 2). L'infécondité générale (toutes femmes) mesurée ainsi (26\% en France) reste cohérente avec celles mesurées dans les autres pays occidentaux : 32 \% pour la Belgique (Brée et al., 2017), 26 \% en Allemagne (Dorbritz et Schwartz, 1996), 23 \% aux Pays-Bas (Rowland, 2007), 24 \% aux États-Unis (Morgan, 1991) et 31 \% en Australie (Rowland, 2007).

(6) C'est Louis Henry qui avait préconisé de rester « à l'échelon national [en se contentant] de classements peu détaillés en grands groupes professionnels ou [...] socioéconomiques » (Henry, 1953, p. 490). 
Quant aux enquêtes Famille, si les taux de non-réponses varient beaucoup d'une enquête à l'autre ${ }^{(7)}$ (Brée et al., 2016b), on obtient des estimations cohérentes des différentes parités et de la descendance finale par cohorte de naissance.

\section{Mesurer la descendance finale et la taille des familles selon la situation matrimoniale des femmes}

La figure 1 regroupe les estimations de la descendance finale des femmes déjà mariées à 45 ans issues des recensements de 1931 et de 1946 et des enquêtes de 1954, 1975, 1982, 1990, 1999 et $2011^{(8)}$.

Figure 1. Descendance finale des femmes déjà mariées par génération selon différentes sources (données d'enquêtes lissées sur trois ans)

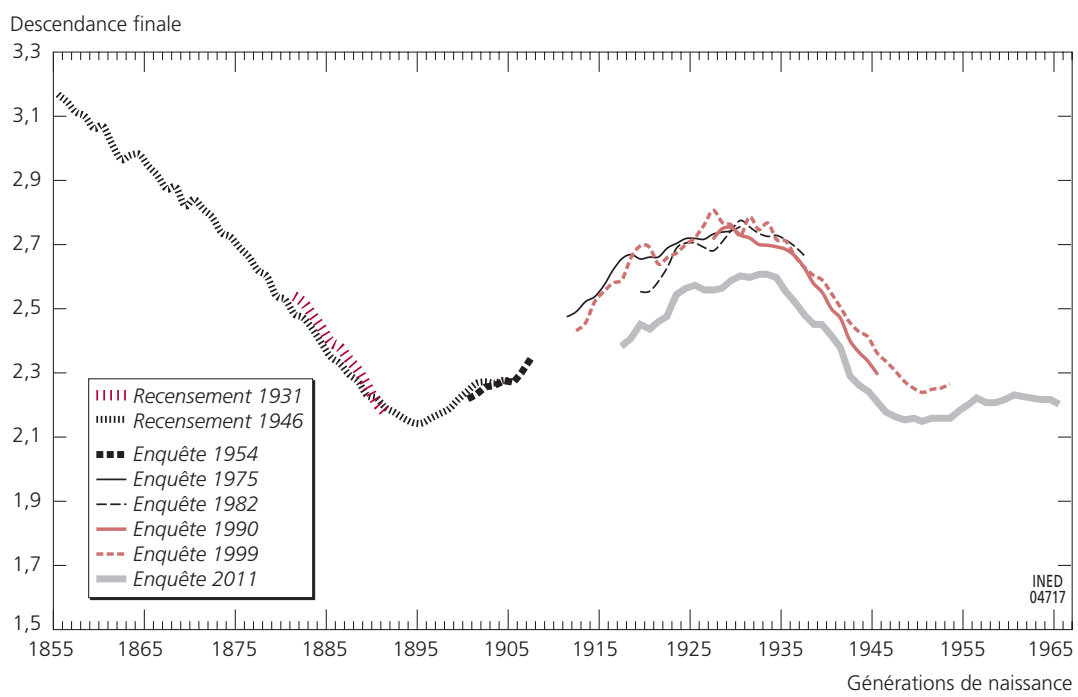

Lecture : Nombre moyen d'enfants nés vivants, par femme mariée, veuve ou divorcée de chaque génération. Sources: Insee, Recensement de la population, 1931, 1946; Insee, ADISP-CMH, enquête Famille, 1954, 1975, 1982, 1990; Insee, ADISP-CMH, Étude de I'histoire familiale, 1999; Insee, ADISP-CMH, Famille et logements, 2011.

Les estimations sont donc très cohérentes, à l'exception toutefois de la dernière enquête Famille (Famille et logements, 2011) dans laquelle les descendances finales sont bien plus faibles que dans les trois précédentes (en particulier celle de 1999). Il semble exister une sous-déclaration des enfants nés avant les unions en cours, en particulier quand ces enfants ne vivent pas avec le couple (Mazuy et Toulemon, 2013).

(7) L'enquête Famille était obligatoire jusqu'en 1990 ; en 1999, l'aspect massivement rétrospectif de l'enquête a conduit ses concepteurs à ne pas demander l'obligation de réponse (Héran, 2005). Cette « contrainte » d'obligation explique très probablement la différence sensible entre les taux de nonréponse des enquêtes de 1982 et 1990 et celles plus récentes de 1999 et 2011.

(8) Pour les enquêtes de 1999 et 2011, les pondérations proposées par les responsables des enquêtes ont été utilisées (poidsm5 pour l'enquête de 1999 et poids_ind pour l'enquête de 2011). 
Qu'en est-il lorsqu'on s'intéresse plus précisément à la distribution du nombre d'enfants (figure 2) ? Concernant l'infécondité des femmes ayant été mariées au moins une fois, le recensement de 1946 est très cohérent avec les données issues des enquêtes de 1954 et 1975, et celle de 1999 pour les générations

Figure 2. Distribution des femmes mariées, veuves ou divorcées par taille des familles (\%) et par génération quinquennale selon différentes sources (données lissées sur trois ans)
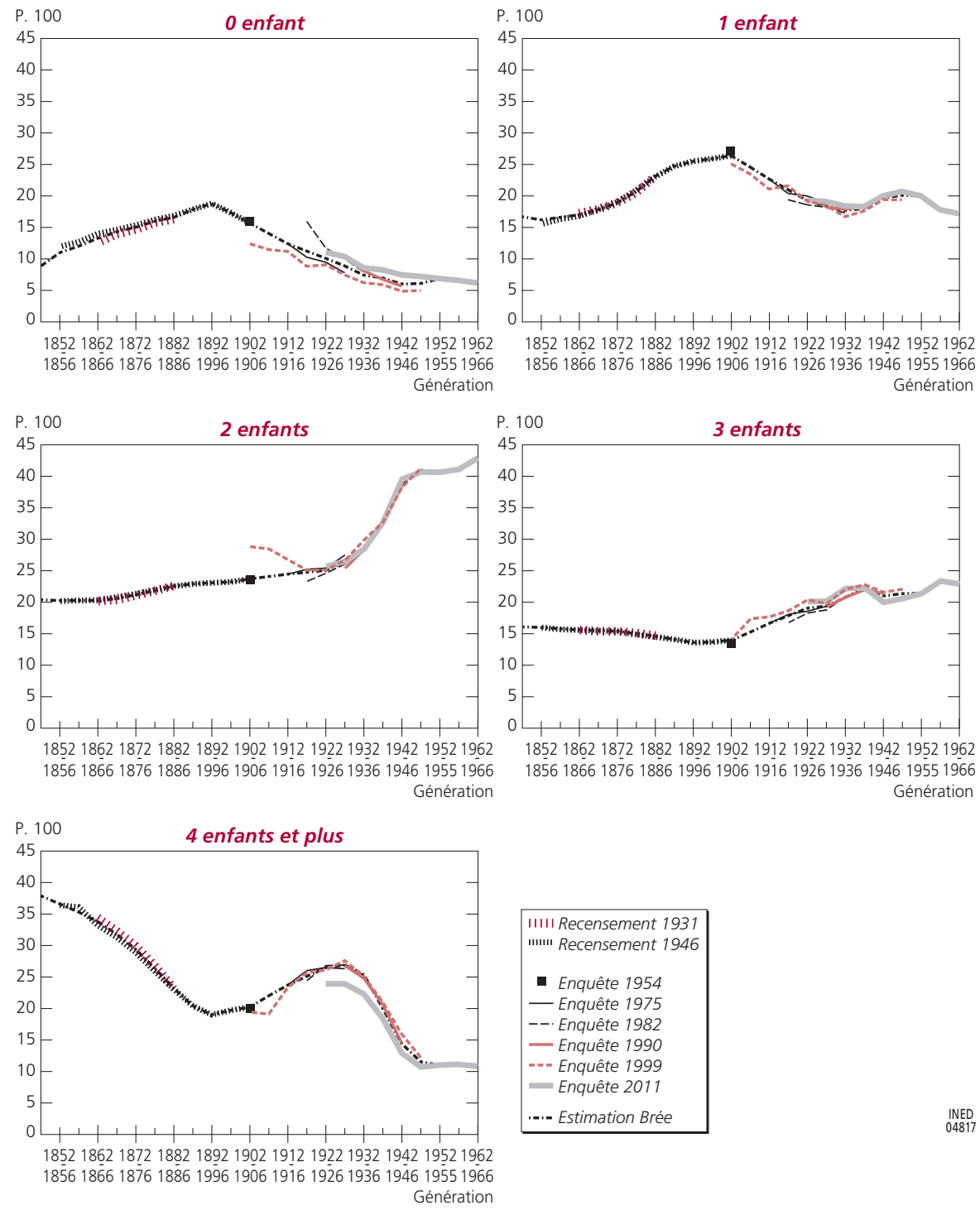

$$
\begin{array}{|l}
\text { IIIII Recensement } 1931 \\
\text { ‥"․ Recensement } 1946 \\
\text { - Enquête } 1954 \\
\text { - Enquête } 1975 \\
\text {--- Enquête } 1982 \\
\text { - Enquête } 1990 \\
\text {--- Enquête } 1999 \\
\text { - Enquête } 2011 \\
\text {-- Estimation Brée }
\end{array}
$$

Lecture : Pour chaque figure : proportion des femmes ayant eu x enfants nés vivants parmi celles mariées, veuves ou divorcées au moins une fois au moment de l'enquête.

Sources : Insee, Recensement de la population, 1931, 1946; Insee, ADISP-CMH, Enquête Famille, 1954, 1975, 1982, 1990; Insee, ADISP-CMH, Étude de I'histoire familiale, 1999; Insee, ADISP-CMH, Famille et logements, 2011. 
nées après 1926. Comparées à ces données, les enquêtes de 1982, 1990 et 2011 surestiment l'infécondité. Pour les catégories « un enfant » et « deux enfants », les données des différentes sources s'ajustent correctement (à l'exception des données de l'enquête de 1999 pour les générations nées avant 1920 des femmes ayant déclaré 2 enfants). Pour les femmes ayant déclaré avoir eu trois enfants, les données sont un peu moins homogènes, mais restent assez cohérentes. En revanche, les mères de quatre enfants et plus sont sous-estimées dans l'enquête de 2011 par rapport aux données des autres enquêtes.

À partir de ces différentes sources, nous fournissons une estimation de la fécondité des femmes d'après les pondérations présentées dans le tableau 2. Les données des statistiques des familles de 1931 et de 1946 ont été utilisées pour les générations nées avant 1906. À partir de la génération 1912-1916 ${ }^{(9)}$, les différentes enquêtes ont été mobilisées en ne tenant pas compte des enquêtes de 1999 et de 2011 pour les générations plus anciennes (ayant au-delà de 75 ans au moment de l'enquête) car les effectifs sont très faibles (figure annexe A.1). Nous avons choisi de conserver ces estimations simples car elles permettent plus facilement de distinguer la fécondité des femmes ayant été mariées de celle ne l'ayant pas été, tout en gardant la cohérence pour les données concernant l'ensemble des femmes. Par ailleurs, ces estimations pourront être actualisées avec les données d'éventuelles prochaines enquêtes Famille. Les estimations de la distribution de la taille des familles et de la descendance finale sont fournies dans le tableau annexe A.1.

Tableau 2. Pondérations utilisées pour les estimations à partir du recensement $(R)$ et des enquêtes Famille $(E)$

\begin{tabular}{|c|c|c|}
\hline Générations <1850 & $\mathrm{R}_{31}$ & Générations 1922-1926 $\quad\left(E_{75}+E_{82}+E_{99}\right) / 3$ \\
\hline Générations 1850-1886 & $\left(R_{31}+R_{46}\right) / 2$ & Générations 1927-1931 $\left(E_{75}+E_{82}+E_{90}+E_{99}\right) / 4$ \\
\hline Générations 1887-1906 & $\mathrm{R}_{46}$ & Générations 1932-1936 $\quad\left(E_{82}+E_{90}+E_{99}\right) / 3$ \\
\hline Générations 1907-1911 & $\begin{array}{l}\text { moyenne des } \\
\text { générations } \\
\text { encadrantes }\end{array}$ & Générations 1937-1946 $\quad\left(E_{90}+E_{99}+E_{2011}\right) / 3$ \\
\hline Générations 1912-1916 & $E_{75}$ & Générations 1947-1951 \\
\hline Générations 1917-1921 & $\begin{array}{l}\text { moyenne des } \\
\text { générations } \\
\text { encadrantes }\end{array}$ & Générations 1952-1966 \\
\hline \multicolumn{3}{|c|}{$\begin{array}{l}\text { Sources: Insee, Recensement de la population, 1931, 1946; Insee, ADISP-CMH, enquête Famille, 1954, 1975, } \\
\text { 1982, 1990; Insee, ADISP-CMH, Étude de I'histoire familiale, 1999; Insee, ADISP-CMH, Famille et logements, } \\
\text { 2011. }\end{array}$} \\
\hline
\end{tabular}

Concernant les femmes jamais mariées à 45 ans, malgré les effectifs réduits, les enquêtes de 1982, 1990, 1999 et 2011 donnent des résultats cohérents quant aux parités et à la descendance finale (figure annexe A.2). Il n'est en revanche

(9) Une moyenne de 1902-1906 et 1912-1916 a été appliquée pour la génération 1907-1911. 
pas possible de mesurer directement la fécondité des femmes jamais mariées pour les générations nées avant 1915. Pour estimer la fécondité générale à partir du recensement de 1946 (qui ne fournit pas de données pour les femmes célibataires), Festy considère que les femmes célibataires contribuent à la fécondité générale à raison de 0,06 première naissance et 0,02 deuxième naissance, soit au total 0,08 naissance pour une descendance finale de 2 à 2,6 naissances pour l'ensemble des femmes (Festy 1979; Ined, 1966, 1991). Cela revient à environ $3 \%$ à $4 \%$ de naissances non légitimées par le mariage, calcul cohérent avec les données sur les naissances illégitimes de Nizard et Maksud (1977). Cependant, cela équivaut à considérer, par exemple pour la génération 1891-1895, que 52 \% des femmes célibataires à 45 ans seraient sans enfant, $32 \%$ auraient eu un enfant, 16 \% deux enfants. Envisager que presque la moitié des femmes célibataires à 45 ans aient eu au moins un enfant paraît un peu excessif, d'autant que, pour les femmes nées en 1912-1916 (premières générations pour lesquelles les parités des femmes célibataires s'alignent avec cohérence sur celles des générations suivantes), ces niveaux sont respectivement de $84 \%$ de femmes célibataires sans enfant, $10 \%$ avec un enfant et $6 \%$ avec deux dans l'enquête de 1999, soit 16 \% de femmes ayant eu au moins un enfant. Nous avons donc plutôt considéré que ces niveaux pouvaient être conservés pour les générations antérieures, au risque de sous-estimer légèrement la contribution des femmes jamais mariées si les naissances illégitimes ont diminué entre ces différentes générations ${ }^{(10)}$. Ces estimations de la fécondité des femmes célibataires à 45 ans sont donc plus faibles que celles de Festy, et par conséquent c'est également le cas pour les estimations de fécondité générale.

La distribution de la taille des familles de l'ensemble des femmes et leur descendance finale est estimée d'après la formule suivante :

$$
\mathrm{Px}_{g}=\mathrm{Px}_{m}^{*}\left(1-\mathrm{C}_{d}\right)+\mathrm{Px}_{c}^{*} \mathrm{C}_{d}
$$

où $\mathrm{Px}_{g}=$ fécondité générale de rang $\mathrm{x}$

$\mathrm{Px}_{m}=$ fécondité des femmes déjà mariées à 45 ans de rang $\mathrm{x}$

$\mathrm{Px}_{c}=$ fécondité des femmes célibataires à 45 ans de rang $\mathrm{x}$

$\mathrm{C}_{d}=$ célibat définitif ${ }^{(11)}$

Les données estimées pour les générations nées à partir des années $1920^{(12)}$ sont très cohérentes avec les données estimées directement à partir des enquêtes (figure annexe A.3). Quant aux estimations pour les générations précédentes,

(10) Les naissances illégitimes sont légèrement plus nombreuses dans les générations nées entre 1860 et 1890 (environ $9 \%$ selon Nizard et Maksud, 1977) que dans celles nées ultérieurement (7\% pour les générations nées vers 1900; 8 \% pour celles nées dans les années 1920; source: Insee, statistiques de l'état civil).

(11) Le niveau de célibat définitif utilisé n'est pas celui des enquêtes (sous-estimé selon Toulemon, 1995), mais celui des recensements de population (Insee). Pour les générations 1850 à 1922-1926, le célibat définitif utilisé est celui publié dans Chasteland et Pressat (1962).

(12) La fécondité de l'ensemble des femmes ne peut pas être estimé pour les générations à partir des recensements de 1931 et de 1946, car seules les femmes mariées, veuves ou divorcées ont été interrogées; c'est ce qui explique le choix de cette méthode. 
elles diffèrent de celles du recensement de 1946 puisque les données disponibles dans ce dernier pour l'ensemble des femmes ont été estimées en considérant que toutes les femmes célibataires n'avaient eu aucun enfant (figure annexe A.4). Elles diffèrent également de celles que l'on peut estimer à partir des données de Depoid (1941) ou de Festy (1979) évaluées à partir de la lecture en diagonale ${ }^{(13)}$ des taux de fécondité par âge (figure annexe A.4). Ces estimations, pour les générations nées avant 1890, sont supérieures à celles que l'on obtient en considérant que toutes les femmes célibataires ont au moins un enfant, ce qui n'est pas vraisemblable : soit la descendance finale calculée de manière rétrospective sous-estime la fécondité, soit elle est surestimée par la méthode de lecture diagonale des taux de fécondité par âge.

Dans la première hypothèse, cela signifierait que les femmes décédées ou ayant migré avaient une fécondité supérieure aux autres. Or, les femmes sorties d'observation ont, en général, une fécondité très légèrement inférieure à celle des survivantes (Andersson et Sobolev, 2013; Brée et al., 2016b). Comme précédemment évoqué, il est également possible que considérer toutes les femmes non répondantes comme n'ayant pas eu d'enfant soit excessif. Il est également possible que, répondant à la question à 45 ans ou plus, certaines femmes aient omis de compter des enfants nés vivants mais décédés après quelques jours, voire quelques heures ${ }^{(14)}$.

Dans la seconde hypothèse, la descendance finale pourrait être surestimée par la lecture diagonale « car les fragments d'histoire des diverses générations que représente chacun de ces taux une année donnée dépendent de l'histoire passée de ces générations » (Sardon, 1990, p. 17).

Le niveau de fécondité des femmes célibataires et de l'ensemble des femmes nées avant 1890 se situe probablement entre les estimations de Festy (probablement surévaluées) et les nôtres (probablement sous-évaluées). Il semble qu'à partir de cette analyse des sources et des différentes estimations qui en découlent, les estimations de la taille des familles des femmes mariées soient les plus robustes, en particulier pour les générations les plus anciennes.

\section{II. Évolution de la taille des familles des générations 1850 à 1966}

\section{1. Évolution du nombre d'enfants par famille}

\section{Les femmes déjà mariées}

La figure 3 présente l'évolution de la taille des familles et de la descendance finale des femmes déjà mariées dans les générations françaises nées entre 1850

(13) Cette méthode consiste à faire la somme des taux par âge se rapportant à une même génération (par exemple, pour la génération née en 1930, on ajoute les taux de fécondité à 20 ans en 1950, à 21 ans en 1951, etc.).

(14) La question du comptage des enfants mort-nés n'intervient pas en revanche car la question porte sur les enfants nés vivants. 
et 1966. Elle montre que des niveaux de fécondité similaires ne résultent pas toujours des mêmes composantes par parité (Toulemon, 2001). Les générations 1897-1901 et 1957-1961 atteignent les mêmes faibles niveaux de descendance finale (2,22 enfants par femme), mais alors que pour les premières le niveau s'explique principalement par une forte proportion de femmes n'ayant eu aucun enfant (17\%) ou un seul (26\%), il est le résultat pour les secondes de la prédominance des familles de deux enfants $(41 \%)^{(15)}$.

Figure 3. Distribution du nombre d'enfants pour les femmes déjà mariées (à 45 ans) par génération

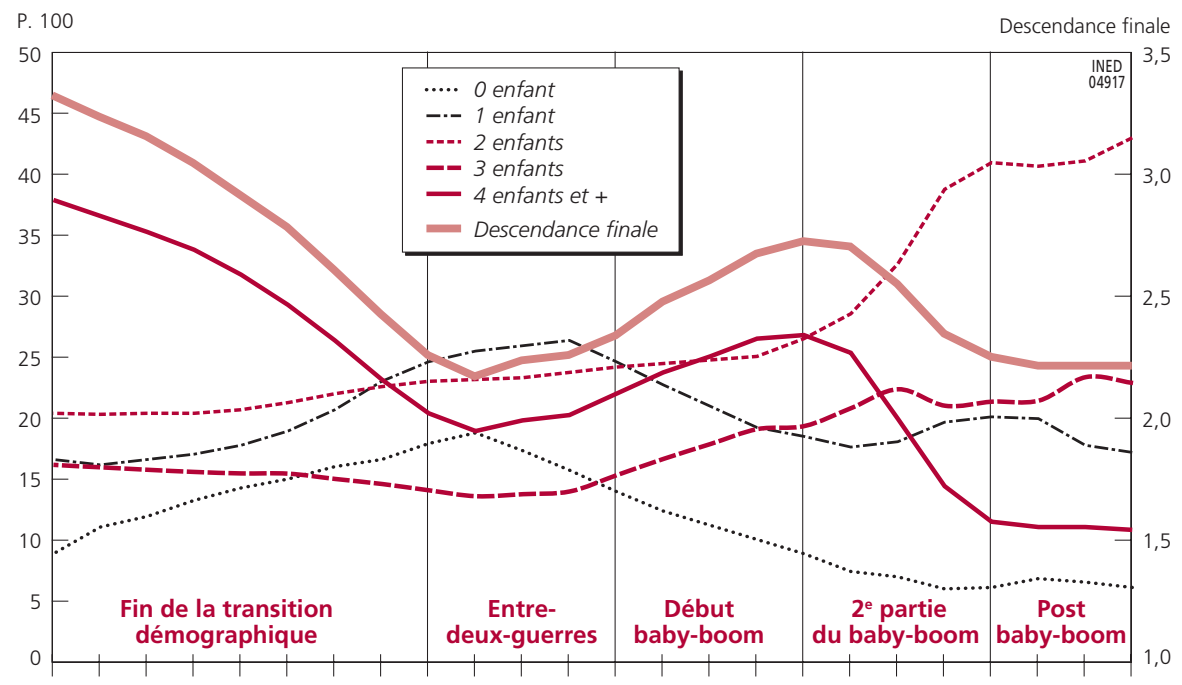

< $\quad 18521857186218671872187718821887189218971902190719121917192219271932193719421947195219571962$ 185118561861186618711876188118861891189619011906191119161921192619311936194119461951195619611966

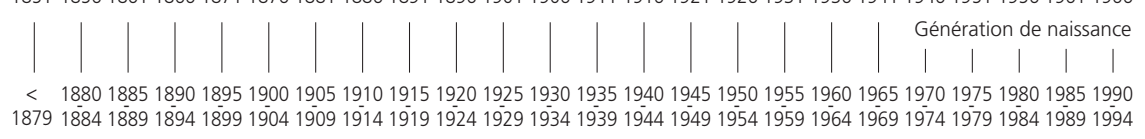

+28 ans*

* L'âge moyen à la naissance est de 28 ans pour les générations les plus anciennes, et de 27 ans pour les plus récentes (Insee, estimations de population et statistiques de l'état civil). Pour plus de facilité, l'âge moyen de 28 ans a été ajouté aux générations de naissance pour donner une idée de la période de fécondité. Source : Tableau annexe A.1.

Cinq groupes de générations apparaissent dans la figure 3. Les générations nées avant 1890 pour lesquelles le modèle dominant (plus de $25 \%$ ) est celui des familles nombreuses de quatre enfants et plus dont l'importance ne cesse cependant de diminuer à la fin du XIX ${ }^{\mathrm{e}}$ siècle (De Luca-Barrusse, 2008); les générations nées entre 1891 et 1911 ayant eu leurs enfants pendant l'entre-deux-guerres pour lesquelles le modèle dominant est l'enfant unique; celles nées entre 1912 et 1931 (ayant eu la majorité de leurs enfants pendant le baby-boom) pour lesquelles on observe une augmentation du nombre de familles de 3 enfants et plus; les

(15) Pour ces deux générations, les mères de 3 enfants ou plus représentent un tiers des femmes. 
générations 1932-1946 dans lesquelles la part des familles de 4 enfants et plus chute alors que l'hégémonie des familles de 2 enfants apparaît (plus de $30 \%$ des familles); et enfin, les générations nées à partir de 1947 pour lesquelles on observe une homogénéisation globale des comportements de fécondité (Daguet, 2002; Prioux, 2002) et une "stagnation des comportements » (Toulemon et al., 2008) qui semble perdurer (Mazuy et al., 2013).

On observe donc un phénomène « cyclique » dans lequel chaque génération s'inscrirait en rupture par rapport à la précédente en adoptant des comportements contraires. Ainsi les filles des femmes ayant eu des descendances nombreuses (générations nées avant 1890) ont mis au monde un nombre réduit d'enfants (générations 1891-1911), les filles de ces dernières engendrant à nouveau des familles nombreuses (générations 1912-1931) alors que leurs petites-filles sont revenues à des fécondités plus basses (générations 1932-1951). Les générations les plus récentes (1952-1966) semblent pourtant conserver un modèle de fécondité principalement caractérisé par un nombre élevé et majoritaire de familles de deux enfants, mais il est difficile de savoir, pour l'instant, s'il s'agit d'une tendance de long terme ou non.

La baisse de la fécondité de la fin du XIX siècle a été interprétée comme le signe d'un changement dans la relation parent-enfant dans laquelle l'affection est désormais au centre (Ariès, 1960, 1980) ; l'enfant devenant " précieux » (Praz, 2005). Le contrôle de la fécondité permettrait d'investir, tant sentimentalement que financièrement, dans la « qualité » des enfants (Alter, 1992; Becker, 1965; Caldwell, 1976). Lanalyse de la taille des fratries montre que ce sont surtout les familles nombreuses (4 enfants et plus) qui sont de moins en moins présentes : leur proportion se réduit de plus de moitié entre les générations 1850 et 1895. Anne-Marie Sohn (1996, p. 809) parle du quatrième enfant comme d'un « chiffre butoir» à partir duquel « on se résout à l'avortement». Pourtant, la diminution de ces familles nombreuses ne conduit pas à une augmentation des familles de trois enfants mais à une augmentation des familles de zéro ou un enfant pour les femmes d'âge fécond pendant l'entre-deux-guerres. Le déclin de la fécondité à partir des générations nées autour de 1850 n'est donc pas caractérisé par une baisse linéaire de toutes les tailles de famille (passage de 4 enfants à trois, de trois à deux etc.) mais par le passage d'un régime dans lequel plus d'un tiers des familles comptaient 4 enfants ou plus à un régime où les familles nombreuses représentent moins d'un cinquième des familles.

L'entre-deux-guerres est une période particulière puisqu'elle est à la fois la fin de la première transition (ou le début de la phase de stagnation si on ne considère le baby-boom que comme un « accident », Desplanques, 1988; Knibiehler, 1997) mais aussi une période de changements socioéconomiques (conséquences démographiques, sociales et économiques de la Première Guerre mondiale et crise économique des années 1930) ${ }^{(16)}$. Malgré les politiques

(16) Sur les liens entre fécondité et crises, voir Eggerickx et al. (2016). 
publiques d'encouragement de la fécondité par la répression ${ }^{(17)}$ et l'incitation ${ }^{(18)}$ visant à reconstruire la population et à combler le déficit des naissances, les femmes qui ont eu leur(s) enfant(s) entre 1920 et 1940 sont parmi les moins fécondes du $\mathrm{XX}^{\mathrm{e}}$ siècle. Il y a donc un décalage entre les lois et les comportements (Cova, 1997), puisque ces lois n'ont eu aucun effet apparent sur la natalité (McLaren, 1996). Si la venue du premier enfant est généralement désirée, les suivants sont plus souvent refusés : la mention de «l'instinct maternel » est fréquente dans les témoignages de joie recueillis par Rebreyend (2003, p. 214), mais elle se raréfie avec les maternités rapprochées, et les grossesses non désirées sont alors assimilées à un piège. Les familles d'un enfant paraissent vraiment être caractéristiques de l'entre-deux-guerres (Brée et al., 2016a, 2017). Certains chercheurs considèrent cette prégnance des familles de taille réduite davantage comme une conséquence des évènements particuliers à cette période que comme un choix (Rowland, 2007). Pour d'autres, l'augmentation de l'infécondité et des familles d'un enfant montre l'accélération de l'acceptation de comportements (notamment être marié et volontairement sans enfant) qui n'étaient pas envisageables auparavant (Anderson, 1998; Morgan, 1991). Ces comportements sont parfois considérés comme pionniers et révélateurs des transformations à venir (Anderson, 1998; Van Bavel et Kok, 2010). Il est malaisé de comprendre ce qui conduit à ces faibles niveaux de fécondité dans l'entredeux-guerres, même si la majorité des théories soulignent, en plus de la continuation du déclin de la fécondité lié à la fin de la transition, l'effet aggravateur du contexte (Brée et al., 2016a).

Qu'en est-il de la remontée observée à partir de la génération 1895 (Festy, 1979) ? Le baby-boom français se caractérise par une baisse de l'infécondité et des familles d'un enfant, une très légère augmentation des familles de deux enfants, mais surtout par une forte augmentation de la proportion de familles de trois enfants et plus. En simulant un déclin linéaire des familles de quatre enfants et plus entre les générations 1897 et 1937 au lieu de la remontée observée, et en redistribuant proportionnellement les autres tailles de famille, la descendance finale stagne comme dans le modèle théorique de la transition démographique (figure annexe A.5). Ce résultat va dans le sens de l'interprétation du baby-boom comme une parenthèse (Coleman, 2004) et établirait l'entre-deux-guerres comme la fin de la transition démographique, ou du moins comme le début d'une longue phase de stagnation de la fécondité moyenne puisque la composition des familles a encore évolué par la suite. Les raisons qui ont amené les générations de l'enfant rare à avoir autant de familles nombreuses font toujours débat au sein des démographes (Calot et Sardon, 1998; Van Bavel et Reher, 2013). Le baby-boom a pu être considéré comme une conséquence de l'optimisme lié à la fin de la guerre mais, la fécondité ayant

(17) Lois 1920 : interdiction de la publicité et de la vente des moyens de contraception.

Loi 1923 : correctionnalisation de l'avortement (transformation du crime en délit, allègement de la sanction judiciaire).

(18) Fête des mères et allocations familiales en 1932 (généralisées en 1939). 
repris avant la fin de la Seconde Guerre mondiale, cette hypothèse a été rejetée (Calot et Sardon, 1998; Van Bavel et Reher, 2013). Les explications économiques ont également souvent été évoquées mais n’ont finalement trouvé que peu d'appui empirique (en dehors des études sur les États-Unis; Doliger, 2008). Pour Van Bavel et Reher (2013), le baby-boom est principalement lié à un boom de nuptialité, à une baisse de l'âge moyen au mariage et, en conséquence, de l'âge moyen à la première maternité. L'allongement des durées de mariage et l'utilisation de moyens contraceptifs toujours traditionnels (principalement le coït interrompu) ont provoqué une augmentation de la taille des familles. Dans le cas spécifique de la France, pour Calot et Sardon (1998) comme pour Chesnais (2006), la politique familiale française a également pu provoquer un « sentiment d'urgence » pour éviter le déclin de la population.

Avec les générations nées au début des années 1930, la fécondité diminue à nouveau. Ce baby-bust est caractérisé, en France, par l'augmentation des familles de 2 enfants et par la baisse des familles de 4 enfants et plus. Le babybust est souvent expliqué par la révolution contraceptive (Westoff et Ryder, 1977), alors qu'il précède de quelques années la diffusion et l'utilisation de la pilule comme contraceptif (mais pas forcément son apparition; Caldwell, 2001). Pour Leridon (1987), il est le résultat combiné d'une diminution du nombre d'enfants désirés et d'une plus grande efficacité de la planification des naissances. Van de Kaa et Lesthaghe (Lesthaghe, 1995; Lesthaghe et Van de Kaa, 1986; Van de Kaa, 1987) ont avancé le concept de « seconde transition démographique " pour décrire les transformations du dernier tiers du $\mathrm{Xx}^{\mathrm{e}}$ siècle ayant mené, entre autres choses, à cette baisse de la fécondité. Selon eux, l'émergence d'un modèle familial individualiste dans lequel les relations de couple sont le socle de la famille, a affecté le processus de formation de la famille (retard de lâge au mariage et augmentation des divorces) ainsi que les comportements reproducteurs, avec un déclin marqué de la fécondité. Coleman (2004), parmi d'autres, a cependant contesté cette théorie, car selon lui le mouvement de la fécondité ne serait qu'une phase dans un processus global de transition de la fécondité entamé depuis le tournant du XX ${ }^{e}$ siècle, voire avant.

Dans les générations les plus récentes (femmes nées à partir des années 1950), la fécondité atteint des niveaux très bas caractérisés par une forte polarisation sur les familles de deux enfants et la plus faible proportion observée de familles nombreuses ( 4 enfants et plus). Pourtant, même si les très faibles niveaux de fécondité observés depuis la fin des années 1970 et surtout dans les années 1980 sont un des éléments caractérisant la « seconde transition démographique ", les niveaux actuels de fécondité ne sont pas exceptionnels et rejoignent les niveaux observés avant le baby-boom (Brée et al., 2017). La prédominance des familles de deux enfants est, en revanche, spécifique aux femmes nées à partir des années 1940. Frejka (2008) pose cependant la question de la durée de cette prédominance et de son éventuel remplacement par les 
familles sans enfant ou avec un seul enfant. En France, l'infécondité se maintient à des niveaux assez faibles par rapport aux autres pays européens, mais les familles n'ayant qu'un seul enfant sont assez nombreuses et leur proportion reste relativement stable depuis les générations nées dans les années 1940. Cette stabilité est d'ailleurs remarquable, étant donné les effets attendus de l'augmentation de l'âge de la mère à la première naissance et le plus fort risque de dissolution de l'union chez les couples avec un enfant qui devraient influer positivement sur la probabilité d'avoir un seul enfant (Breton et Prioux, 2009).

\section{Les femmes n'ayant jamais été mariées}

Si les femmes qui ne se sont jamais mariées représentent une proportion assez faible de l'ensemble des femmes dans les générations étudiées ( $7 \%$ à $12 \%$ ), leurs comportements féconds méritent d'être analysés, d'autant plus que leur poids croît parmi les générations les plus récentes et, de ce fait, le poids de leurs naissances dans la fécondité générale ${ }^{(19)}$.

Alors que les femmes nées avant la Première Guerre mondiale avaient très peu d'enfants hors mariage -l'illégitimité étant encore considéré comme un comportement déviant (Fauve-Chamoux et Brunet, 2014)-, les comportements évoluent après la Seconde Guerre mondiale. Les femmes nées à partir de la fin des années 1920 et du début des années 1930 jamais mariées ont de plus en plus souvent au moins un enfant (figure 4), et ce phénomène s'amplifie dans les générations suivantes. Si les explications du baby-boom sont souvent tournées vers la hausse de la fécondité légitime et de l'âge au mariage (Van Bavel et Reher, 2013), il apparaît néanmoins que les femmes jamais mariées contribuent également, en France du moins, à l'augmentation de la fécondité.

À partir des années 1970, l'augmentation de la cohabitation avant le mariage (Roussel, 1978) et celle de l'union libre (Guibert-Lantoine et al., 1994; Rault et Régnier-Loilier, 2015) font du mariage un acte de moins en moins nécessaire à la constitution des familles. Les naissances hors mariage se banalisent (Muñoz-Perez et Prioux, 1999) même si le mariage est resté un préalable à la procréation et majoritaire jusqu'en 2007, date à partir de laquelle la proportion d'enfants nés hors mariage devient supérieure à celle des enfants nés d'un couple marié (Insee, statistiques de l'état civil). Parmi les femmes jamais mariées, un grand nombre a cependant eu des relations de couple, souvent cohabitantes voire officialisées civilement (pacs depuis 1999) (Rault et RégnierLoilier, 2015), mais ces dernières ont toujours moins d'enfants que les autres. Dans la génération née en 1962-1966, les femmes jamais mariées sont beaucoup plus nombreuses à n'avoir aucun enfant $(40 \%)$ que les femmes déjà mariées (6\%). Cependant, si on ne tient compte que des femmes ayant eu au moins un enfant, les comportements sont plus proches. Les mères jamais mariées ont plus souvent un seul enfant que les mères déjà mariées (39\% contre $19 \%$ ), mais elles ont presque aussi souvent deux enfants que les autres ( $40 \%$ contre

(19) Sur le poids de la fécondité illégitime dans la fécondité générale au XIX ${ }^{e}$ siècle, voir Brée (2014). 
Figure 4. Distribution du nombre d'enfants pour les femmes célibataires à 45 ans, par génération

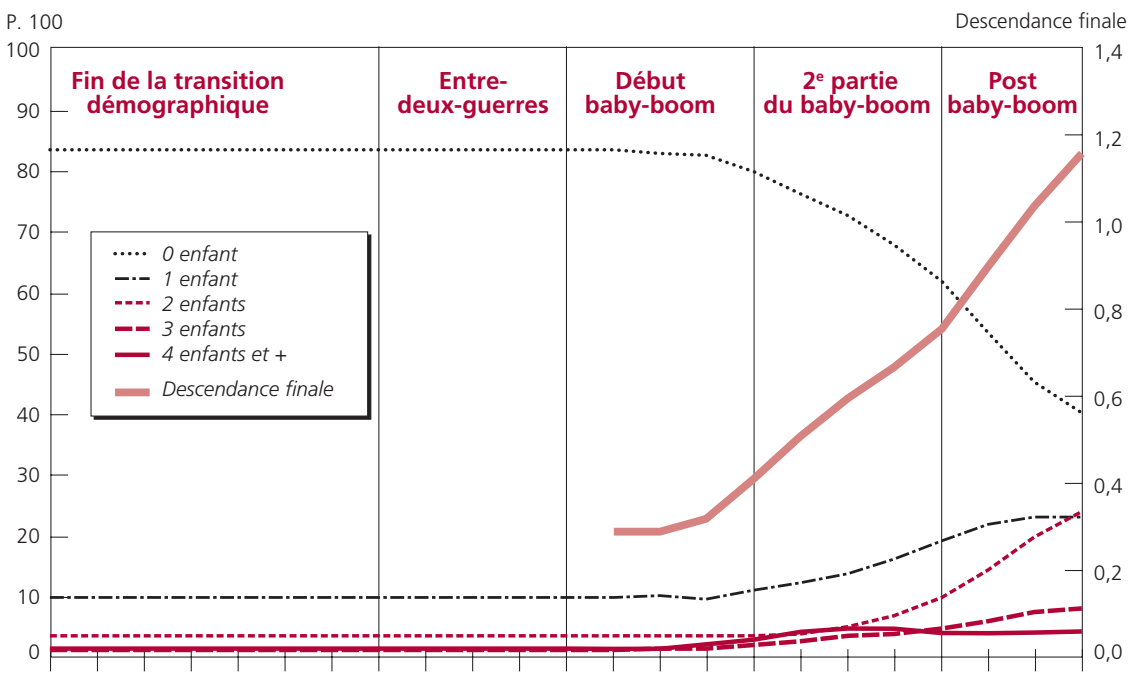

18521857186218671872187718821887189218971902190719121917192219271932193719421947195219571962 $18 \overline{5} 18 \overline{6} 118 \overline{6} 18 \overline{7} 118 \overline{7} 618 \overline{81} 18 \overline{86} 18 \overline{9} 118961901190619 \overline{1} 19 \overline{16} 19 \overline{21} 192619311936194119 \overline{4} 1951195619611966$

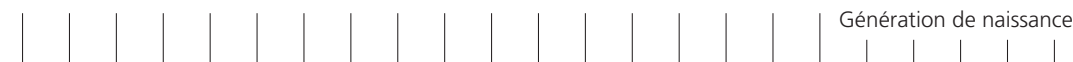

18801885189018951900190519101915192019251930193519401945195019551960196519701975198019851990

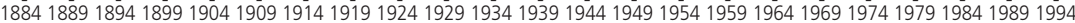

+28 ans*

* L'âge moyen à la naissance est de 28 ans pour les générations les plus anciennes, et de 27 ans pour les plus récentes (Insee, estimations de population et statistiques de l'état civil). Pour plus de facilité, l'âge moyen de

28 ans a été ajouté aux générations de naissance pour donner une idée de la période de fécondité.

Note : Pour les générations les plus anciennes (jusqu'à la génération 1912-1916) la distribution est la même puisque nous leur avons appliqué celle de la génération 1912-1916. Même si le mouvement de la distribution entre les générations nées entre 1855 et 1910 n'est probablement pas aussi constant, le peu de changement observé entre les générations 1912-1916 et 1922-1926 semble valider notre choix méthodologique.

Source : Tableau annexe A.1.

$44 \%)$. Ces différences de fécondité peuvent s'expliquer par le fait qu'un certain nombre de ces femmes n'ont pas de compagnon, ou du moins pas au moment où elles auraient pu avoir des enfants, mais aussi car les femmes qui ne souhaitent pas d'enfant vont bien moins souvent se tourner vers le mariage que celles qui en désirent.

\section{L'ensemble des femmes}

La taille des familles pour l'ensemble des femmes (figure 5) est évidemment très proche de celle des femmes déjà mariées, celles-ci étant largement majoritaires ( $88 \%$ à $93 \%$ des femmes). Les variations principales s'observent pour l'infécondité et les parités « un enfant » puisque ce sont les parités les plus concernées par le comportement des femmes célibataires. L'évolution de la descendance finale apparaît déterminée par celle des familles nombreuses, encore davantage que pour les seules femmes mariées. 
Figure 5. Distribution du nombre d'enfants pour l'ensemble des femmes, par génération (1850-1966)

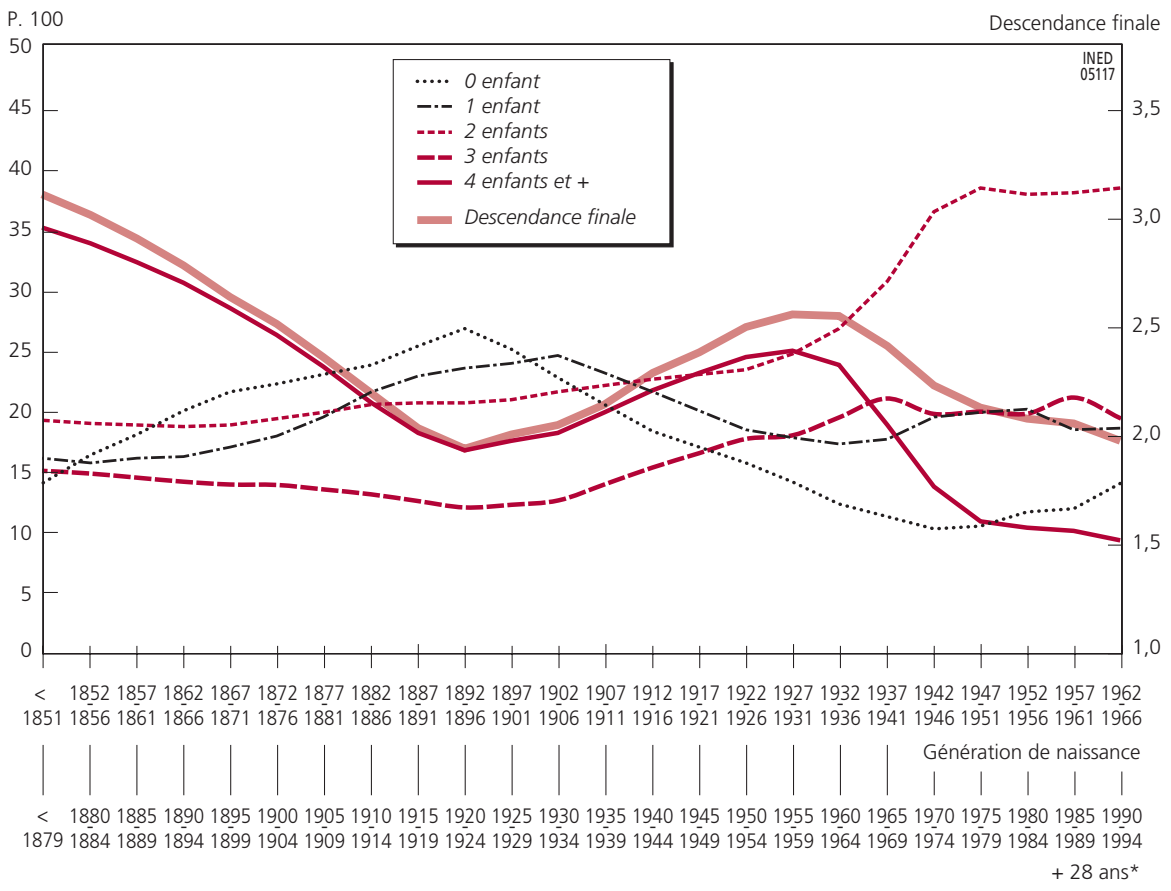

* L'âge moyen à la naissance est de 28 ans pour les générations les plus anciennes, et de 27 ans pour les plus récentes (Insee, estimations de population et statistiques de l'état civil). Pour plus de facilité, l'âge moyen de 28 ans a été ajouté aux générations de naissance pour donner une idée de la période de fécondité. Source : Tableau annexe A.1.

L'analyse de l'infécondité doit donc toujours être menée avec attention puisque les niveaux varient fortement selon qu'on prend en compte l'ensemble des femmes ou les femmes déjà mariées. Pour la France, l'infécondité des femmes nées en 1892-1896 s'élève à 26,9 \% pour l'ensemble des femmes et à 18,8 \% pour les seules femmes déjà mariées.

S'il peut sembler plus rigoureux d'analyser la fécondité des femmes déjà mariées (les données sont meilleures et l'indicateur plus solide puisque les femmes déjà mariées sont a priori toutes soumises au risque de grossesse), il est important pour les générations les plus récentes de considérer la fécondité générale, puisqu'il n'existe plus de distinction entre les enfants nés hors mariage et ceux nés dans le mariage.

\section{Les probabilités d'agrandissement des familles}

En complément de la taille des familles qui indique combien de femmes ont eu exactement un enfant, deux enfants etc. au cours de leur vie, les probabilités d'agrandissement mesurent la part de femmes passées d'un à deux 
enfants, de deux à trois et ainsi de suite (figure 6). La probabilité $\mathrm{a}_{0}$ exprime, pour toutes les femmes sans enfant, le risque d'avoir au moins une naissance (son complément à $1\left(1-a_{0}\right)$ représente le risque de rester inféconde). La probabilité $\mathrm{a}_{1}$ exprime, pour toutes les femmes ayant déjà au moins un enfant, le risque d'en avoir au moins un deuxième; et ainsi de suite. L'intérêt de cet indicateur est de savoir combien de femmes sont mères d'au moins un enfant, au moins deux, etc., sans tenir compte des naissances ultérieures. Ainsi, il est étonnant d'observer que la probabilité d'avoir un deuxième enfant est au même niveau dans les générations nées vers 1960 que dans celles nées cent ans plus tôt alors que les générations les plus récentes ont deux fois plus souvent des familles de deux enfants (39\%) que les générations nées vers 1860 (18\%).

Figure 6. Probabilités d'agrandissement pour l'ensemble des femmes nées entre 1850 et 1966

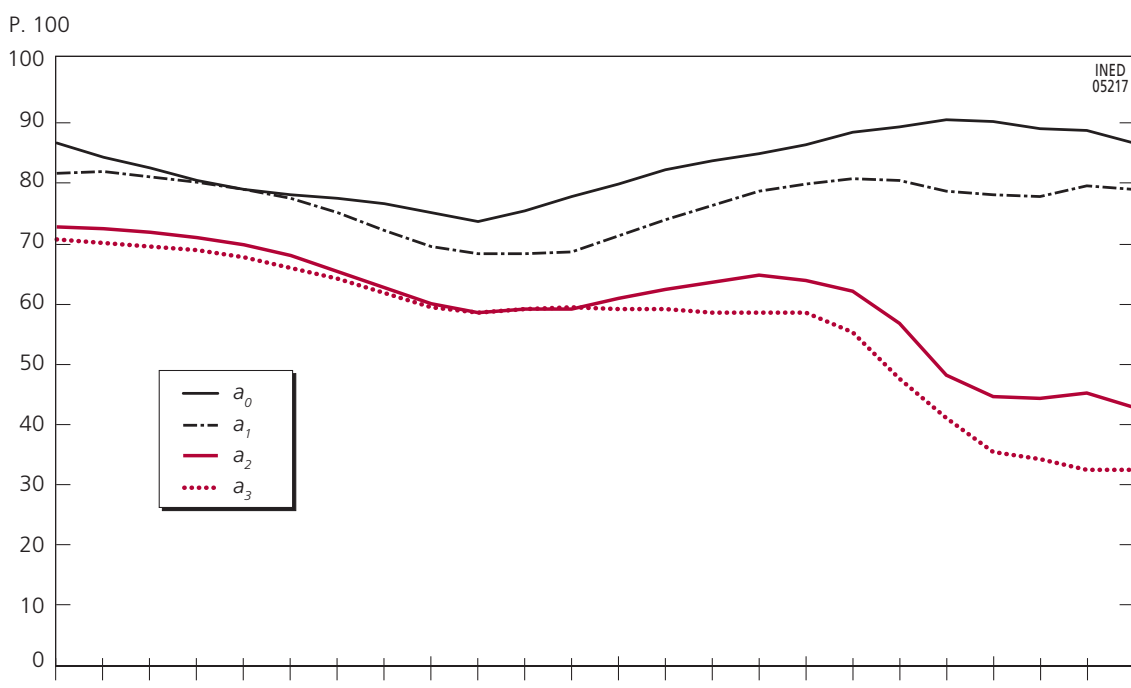

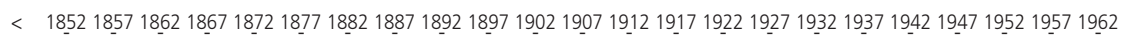

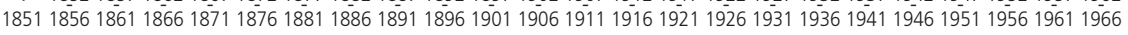

18801885189018951900190519101915192019251930193519401945195019551960196510 187918841889189418991904190919141919192419291934193919441949195419591964196919741979198419891994

+28 ans*

* L'âge moyen à la naissance est de 28 ans pour les générations les plus anciennes, et de 27 ans pour les plus récentes (Insee, estimations de population et statistiques de l'état civil). Pour plus de facilité, l'âge moyen de 28 ans a été ajouté aux générations de naissance pour donner une idée de la période de fécondité. Lecture : Dans les générations nées en 1892-1896, la probabilité d'avoir un premier enfant s'élevait à 73\% $\left(a_{0}\right)$, celle d'en avoir un deuxième à $68 \%\left(a_{1}\right)$ et ainsi de suite.

Note : Les probabilités d'agrandissements pour les générations nées après 1962 sont données à titre indicatif. Source : Enquête Famille et logements (2011).

Ce qui apparaît en premier lieu est une diminution de toutes les probabilités d'agrandissement pour les générations de femmes nées entre 1850 et 1891. Elle est suivie d'une rupture à partir des générations 1892 et 1911 qui ont eu leurs 
enfants pendant l'entre-deux-guerres : leur probabilité d'avoir un premier enfant s'accroît. On observe l'amorce d'une augmentation des probabilités d'avoir un deuxième enfant dès la génération 1902-1906 et un troisième pour la suivante. À partir des générations nées en 1927-1931, la probabilité d'avoir un premier enfant puis un second est plus élevé que dans les générations nées au milieu du XIX ${ }^{e}$ siècle; en revanche, les probabilités supérieures chutent, indiquant une rupture dans les comportements reproductifs. Les choix de fécondité sont alors de plus en plus dépendants du nombre d'enfants déjà nés, ce qui est un élément caractéristique des régimes de fécondité plus contrôlée (Festy, 1979). Enfin, à partir des femmes nées dans les années 1950, qui ont leurs enfants à partir du début des années 1980, les comportements ont tendance à stagner : $87 \%$ des femmes ont eu au moins un enfant, et elles ont la plus forte probabilité d'en avoir un deuxième (78\%). Elles sont en revanche très peu à en avoir eu plus de deux : les probabilités d'avoir un troisième enfant $\left(\mathrm{a}_{2}=44 \%\right)$ ou plus $\left(\mathrm{a}_{3}=33 \%\right)$ se sont effondrées. La stagnation des probabilités $a_{2}$ et $a_{3}$ à partir des générations nées après la Seconde Guerre mondiale peut indiquer un effet de polarisation de certaines femmes sur des descendances nombreuses. Les familles nombreuses sont plus rares que dans les générations précédentes mais traduisent sans doute le choix d'une moindre limitation. Il est possible qu'il s'agisse d'une conséquence de l'immigration internationale et de l'installation durable de communautés à fécondité plus élevée que la moyenne française (Masson, 2013).

Si les commentaires précédents sont également valables pour les femmes ayant déjà été mariées (figure annexe A.6), les probabilités d'agrandissement des femmes célibataires ont une évolution bien différente (figure 7). Tout d'abord, la probabilité d'avoir un (premier) enfant passe de $17 \%$ à $60 \%$ entre les femmes jamais mariées nées entre le début des années 1920 et la fin des années 1960. Mais surtout, la probabilité d'avoir un second enfant ne cesse d'augmenter entre ces générations, à tel point que les femmes jamais mariées nées dans les années 1960 et mères d'un premier enfant en ont quasiment toutes eu un second. Elles ne sont, en revanche, que $35 \%$ à avoir un troisième enfant puis, parmi celles-ci, la même proportion à en avoir un quatrième. Si les femmes jamais mariées ont toujours des probabilités d'avoir un premier et un second enfant bien plus faibles que les femmes mariées ou qui l'ont été, leurs comportements ressemblent pourtant de plus en plus à ceux des femmes déjà mariées, en particulier pour celles qui ont déjà deux enfants; les femmes qui ont déjà trois enfants ont ainsi les mêmes probabilités d'en avoir un supplémentaire que les femmes déjà mariées. 
Figure 7. Probabilités d'agrandissement

pour les femmes jamais mariées nées entre 1850 et 1966

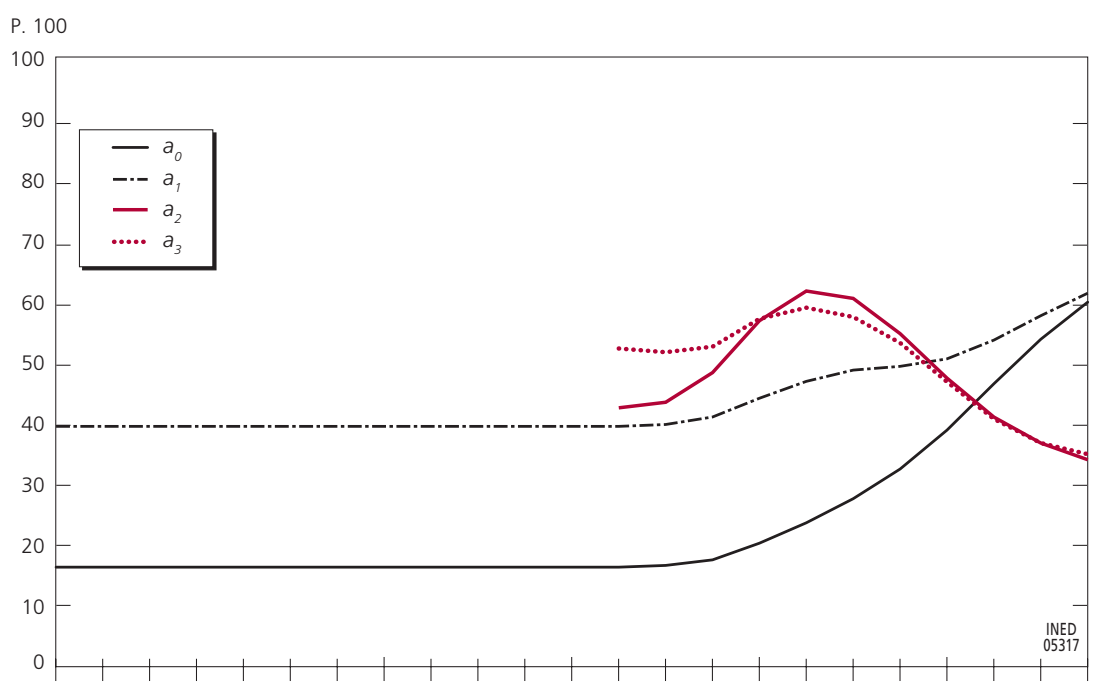

< 1857186218671872187718821887189218971902190719121917192219271932193719421947195219571962 $18571861186618 \overline{7} 1187618 \overline{10} 18861891189619011906191119161921192619311936194119461951195619611966$

< 1885189018951900190519101915192019251930193519401945195019551960196519701975198019851990

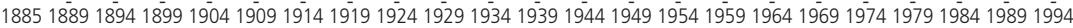

$$
+28 \text { ans* }
$$

* L'âge moyen à la naissance est de 28 ans pour les générations les plus anciennes, et de 27 ans pour les plus
récentes (Insee, estimations de population et statistiques de l'état civil). Pour plus de facilité, I'âge moyen de
28 ans a été ajouté aux générations de naissance pour donner une idée de la période de fécondité. Source : Enquête Famille et logements (2011).

\section{Conclusion}

Malgré les difficultés liées à la pluralité des sources disponibles et l'hétérogénéité des résultats, il est possible d'évaluer l'évolution de la distribution du nombre d'enfants que les femmes nées entre 1850 et 1966 ont mis au monde au cours de leur vie. Cette analyse sur la longue durée des parités et des probabilités d'agrandissement nuance les conclusions que l'on pourrait simplement tirer de la lecture des moyennes (descendances finales) et encourage à toujours prendre en compte la distribution des parités dans l'analyse de la fécondité (Anderson, 1998). La diminution considérable des familles nombreuses (4 enfants et plus) pour les générations nées entre 1850 et 1900, l’importance de l'infécondité et des familles d'un enfant dans les générations ayant eu leurs enfants pendant l'entre-deux-guerres, ou la très forte augmentation des familles de deux enfants à partir des générations nées dans le courant des années 1920, apparaissent ainsi clairement. La prise en compte de la taille des familles est donc importante pour l'analyse de la fécondité puisqu'elle montre qu'à 
descendance finale égale, la distribution des parités peut être très variable, révélant des conceptions différentes de la famille. Elle est notamment importante pour l'analyse historique de la famille ou de la place des enfants et des femmes dans la société. L'analyse sur le long terme est également essentielle pour mettre en perspective et comprendre l'évolution récente de la fécondité. En effet, si elle atteint de très faibles niveaux, ils ne sont pourtant pas exceptionnels à l'échelle d'un siècle de fécondité des générations, et ce ne sont pas tant les basses fécondités que l'uniformatisation vers la famille à deux enfants qui caractérise la fécondité des générations les plus récentes.

Cette recherche souligne enfin l'intérêt de la différenciation de la fécondité de l'ensemble des femmes, des femmes ayant été mariées et des femmes célibataires, notamment dans l'analyse de l'infécondité et des basses fécondités (1 enfant). Celle-ci confirme le poids de la fécondité des femmes déjà mariées jusqu'aux générations nées dans les années 1920 et celui de la transformation de la mise en couple sur la fécondité depuis.

Le renouveau des recherches sur la fécondité au $\mathrm{xx}^{\mathrm{e}}$ siècle ${ }^{(20)}$ indique l'intérêt que revêt toujours la compréhension de la fin de la transition démographique (Brée et al., 2016a, 2016c; Breschi et al., 2016), du baby-boom (Duvoisin et al., 2016; Gauvreau et Laplante, 2016) et du baby-bust (Nomès et Van Bavel, 2016). L'analyse rétrospective de la composition des familles en fonction des niveaux d'instruction ou selon l'appartenance religieuse et en tenant compte de la nuptialité apporte de nouveaux éléments à la compréhension du mouvement de la fécondité, tout comme l'approche infranationale qui permet de mieux analyser l'hétérogénéité des comportements, comme le préconisaient Van Bavel et Reher (2013). Lapport des approches qualitatives (Bonvalet et al., 2011; Rebreyend, 2003; Rusterholz, 2015, 2017; Sohn, 1996) est aussi de plus en plus souvent souligné, et il semble évident que la compréhension du mouvement de la fécondité au $\mathrm{XX}^{\mathrm{e}}$ siècle et jusqu'à nos jours ne saurait se faire sans ces approches croisées.

(20) Voir par exemple le numéro 2016-2 des Annales de démographie historique. 
ANNEXES 
Tableau A.1. Distribution du nombre d'enfants par femme (\%) et descendance finale

\begin{tabular}{|l|c|c|c|c|c|c|}
\hline Générations & 0 enfant & 1 enfant & 2 enfants & 3 enfants & $\begin{array}{c}4 \text { enfants } \\
\text { et }+\end{array}$ & $\begin{array}{c}\text { Descendance } \\
\text { finale }\end{array}$ \\
\hline \multicolumn{7}{|c|}{ Ensemble des femmes } \\
\hline Avant 1857 & 19,1 & 15,5 & 18,5 & 14,3 & 32,7 & 2,91 \\
$1857-1861$ & 20,1 & 15,9 & 18,5 & 14,1 & 31,5 & 2,83 \\
$1862-1866$ & 21,1 & 16,2 & 18,5 & 14,0 & 30,2 & 2,75 \\
$1867-1871$ & 22,2 & 16,9 & 18,7 & 13,8 & 28,3 & 2,62 \\
$1872-1876$ & 22,7 & 17,9 & 19,3 & 13,8 & 26,2 & 2,51 \\
$1877-1881$ & 23,1 & 19,6 & 20,0 & 13,6 & 23,8 & 2,37 \\
$1882-1886$ & 23,9 & 21,6 & 20,6 & 13,1 & 20,8 & 2,20 \\
$1887-1891$ & 25,5 & 23,0 & 20,7 & 12,6 & 18,2 & 2,03 \\
$1892-1896$ & 26,9 & 23,6 & 20,7 & 12,0 & 16,8 & 1,94 \\
$1897-1901$ & 25,2 & 24,0 & 21,0 & 12,3 & 17,6 & 2,00 \\
$1902-1906$ & 22,8 & 24,7 & 21,6 & 12,6 & 18,2 & 2,05 \\
$1907-1911$ & 20,6 & 23,2 & 22,2 & 14,0 & 20,0 & 2,15 \\
$1912-1916$ & 18,4 & 21,7 & 22,8 & 15,4 & 21,8 & 2,29 \\
$1917-1921$ & 17,0 & 20,1 & 23,1 & 16,6 & 23,2 & 2,39 \\
$1922-1926$ & 15,7 & 18,5 & 23,4 & 17,7 & 24,6 & 2,50 \\
$1927-1931$ & 14,2 & 17,9 & 24,8 & 18,0 & 25,1 & 2,56 \\
$1932-1936$ & 12,3 & 17,3 & 26,9 & 19,6 & 23,9 & 2,55 \\
$1937-1941$ & 11,3 & 17,8 & 30,9 & 21,1 & 19,0 & 2,42 \\
$1942-1946$ & 10,3 & 19,5 & 36,6 & 19,8 & 13,8 & 2,24 \\
$1947-1951$ & 10,6 & 20,0 & 38,6 & 20,0 & 10,9 & 2,13 \\
$1952-1956$ & 11,6 & 20,2 & 38,0 & 19,8 & 10,3 & 2,08 \\
$1957-1961$ & 11,9 & 18,5 & 38,3 & 21,2 & 10,1 & 2,06 \\
$1962-1966$ & 14,1 & 18,6 & 38,6 & 19,4 & 9,3 & 1,97 \\
\hline
\end{tabular}

Femmes mariées, veuves ou divorcées au moment de l'enquête ou du recensement

\begin{tabular}{|l|r|l|l|l|l|l|}
\hline 1850 et avant & 8,8 & 16,7 & 20,5 & 16,1 & 37,9 & 3,33 \\
$1851-1855$ & 11,0 & 16,2 & 20,3 & 16,0 & 36,6 & 3,24 \\
$1857-1861$ & 12,0 & 16,6 & 20,3 & 15,8 & 35,3 & 3,16 \\
$1862-1866$ & 13,3 & 17,0 & 20,3 & 15,6 & 33,8 & 3,05 \\
$1867-1871$ & 14,3 & 17,8 & 20,7 & 15,5 & 31,8 & 2,92 \\
$1872-1876$ & 15,0 & 18,9 & 21,3 & 15,4 & 29,4 & 2,79 \\
$1877-1881$ & 16,0 & 20,7 & 22,0 & 15,0 & 26,4 & 2,61 \\
$1882-1886$ & 16,6 & 23,1 & 22,6 & 14,6 & 23,2 & 2,43 \\
$1887-1891$ & 17,8 & 24,7 & 23,0 & 14,1 & 20,4 & 2,26 \\
$1892-1896$ & 18,8 & 25,5 & 23,2 & 13,6 & 19,0 & 2,17 \\
$1897-1901$ & 17,3 & 25,9 & 23,3 & 13,7 & 19,7 & 2,24 \\
$1902-1906$ & 15,6 & 26,4 & 23,8 & 14,0 & 20,2 & 2,26 \\
$1907-1911$ & 14,0 & 24,6 & 24,1 & 15,3 & 22,0 & 2,34 \\
$1912-1916$ & 12,4 & 22,7 & 24,5 & 16,7 & 23,7 & 2,48 \\
$1917-1921$ & 11,2 & 21,0 & 24,8 & 17,9 & 25,1 & 2,57 \\
$1922-1926$ & 10,0 & 19,3 & 25,1 & 19,1 & 26,5 & 2,68 \\
$1927-1931$ & 8,8 & 18,4 & 26,5 & 19,4 & 26,9 & 2,73 \\
$1932-1936$ & 7,4 & 17,7 & 28,6 & 20,8 & 25,4 & 2,71 \\
$1937-1941$ & 6,9 & 18,0 & 32,7 & 22,4 & 20,0 & 2,55 \\
$1942-1946$ & 6,0 & 19,7 & 38,8 & 21,0 & 14,4 & 2,35 \\
$1947-1951$ & 6,1 & 20,1 & 41,1 & 21,4 & 11,5 & 2,25 \\
$1952-1956$ & 6,8 & 20,0 & 40,7 & 21,4 & 11,0 & 2,22 \\
$1957-1961$ & 6,5 & 17,8 & 41,2 & 23,4 & 11,1 & 2,22 \\
$1962-1966$ & 6,1 & 17,2 & 43,0 & 22,9 & 10,8 & 2,22 \\
\hline
\end{tabular}


Tableau A.1 (suite). Distribution du nombre d'enfants par femme (\%) et descendance finale

\begin{tabular}{|c|c|c|c|c|c|c|}
\hline Générations & 0 enfant & 1 enfant & 2 enfants & 3 enfants & $\begin{array}{c}4 \text { enfants } \\
\text { et }+\end{array}$ & $\begin{array}{c}\text { Descendance } \\
\text { finale }\end{array}$ \\
\hline
\end{tabular}

Femmes célibataires au moment du recensement

\begin{tabular}{|l|l|l|l|l|l|l|}
\hline Avant 1857 & 83,55 & 9,95 & 3,74 & 1,31 & 1,44 & 0,30 \\
$1857-1861$ & 83,55 & 9,95 & 3,74 & 1,31 & 1,44 & 0,30 \\
$1862-1866$ & 83,55 & 9,95 & 3,74 & 1,31 & 1,44 & 0,30 \\
$1867-1871$ & 83,55 & 9,95 & 3,74 & 1,31 & 1,44 & 0,30 \\
$1872-1876$ & 83,55 & 9,95 & 3,74 & 1,31 & 1,44 & 0,30 \\
$1877-1881$ & 83,55 & 9,95 & 3,74 & 1,31 & 1,44 & 0,30 \\
$1882-1886$ & 83,55 & 9,95 & 3,74 & 1,31 & 1,44 & 0,30 \\
$1887-1891$ & 83,55 & 9,95 & 3,74 & 1,31 & 1,44 & 0,30 \\
$1892-1896$ & 83,55 & 9,95 & 3,74 & 1,31 & 1,44 & 0,30 \\
$1897-1901$ & 83,55 & 9,95 & 3,74 & 1,31 & 1,44 & 0,30 \\
$1902-1906$ & 83,55 & 9,95 & 3,74 & 1,31 & 1,44 & 0,30 \\
$1907-1911$ & 83,55 & 9,95 & 3,74 & 1,31 & 1,44 & 0,30 \\
$1912-1916$ & 83,55 & 9,95 & 3,74 & 1,31 & 1,44 & 0,30 \\
$1917-1921$ & 83,44 & 10,01 & 3,74 & 1,36 & 1,45 & 0,29 \\
$1922-1926$ & 82,51 & 10,39 & 3,72 & 1,63 & 1,74 & 0,32 \\
$1927-1931$ & 79,91 & 11,19 & 3,78 & 2,16 & 2,97 & 0,41 \\
$1932-1936$ & 76,41 & 12,49 & 4,10 & 2,83 & 4,17 & 0,51 \\
$1937-1941$ & 72,50 & 14,07 & 5,22 & 3,47 & 4,74 & 0,60 \\
$1942-1946$ & 67,75 & 16,43 & 7,09 & 4,03 & 4,70 & 0,67 \\
$1947-1951$ & 61,49 & 19,25 & 10,20 & 4,84 & 4,22 & 0,76 \\
$1952-1956$ & 53,49 & 21,75 & 14,68 & 5,98 & 4,09 & 0,90 \\
$1957-1961$ & 45,80 & 22,94 & 19,77 & 7,29 & 4,19 & 1,04 \\
$1962-1966$ & 40,17 & 23,27 & 24,10 & 8,10 & 4,36 & 1,16 \\
\hline
\end{tabular}

Source: Calculs de l'auteure à partir de : Insee, Recensement de la population, 1931, 1946 ; Insee, ADISP-CMH, enquête Famille, 1954, 1975, 1982, 1990 ; Insee, ADISP-CMH, Étude de I'histoire familiale, 1999 ; Insee, ADISP-CMH, Famille et logements, 2011. 
Figure A.1. Distribution du nombre de femmes interrogées ayant 45 ans et plus au moment de l'enquête, par génération quinquennale

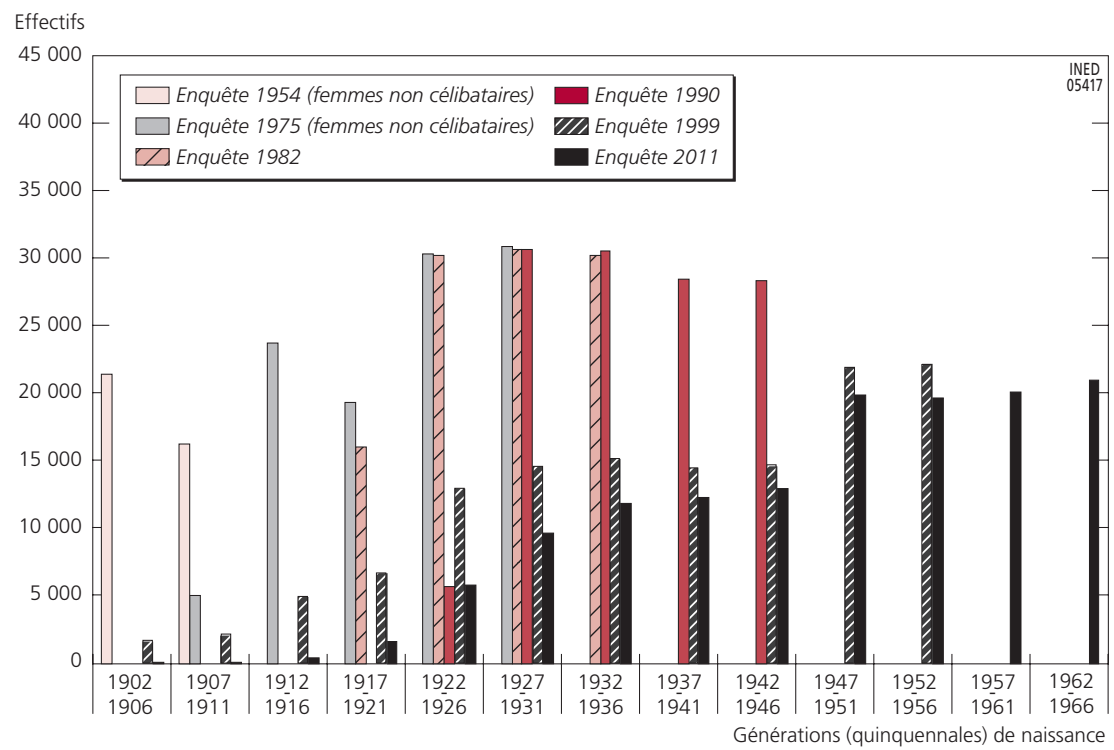

Sources : Insee, ADISP-CMH, enquête Famille, 1954, 1975, 1982, 1990 ; Insee, ADISP-CMH, Étude de I'histoire familiale, 1999; Insee, ADISP-CMH, Famille et logements, 2011. 
Figure A.2. Distribution des parités pour les femmes jamais mariées (générations 1912-1916 à 1962-1966)
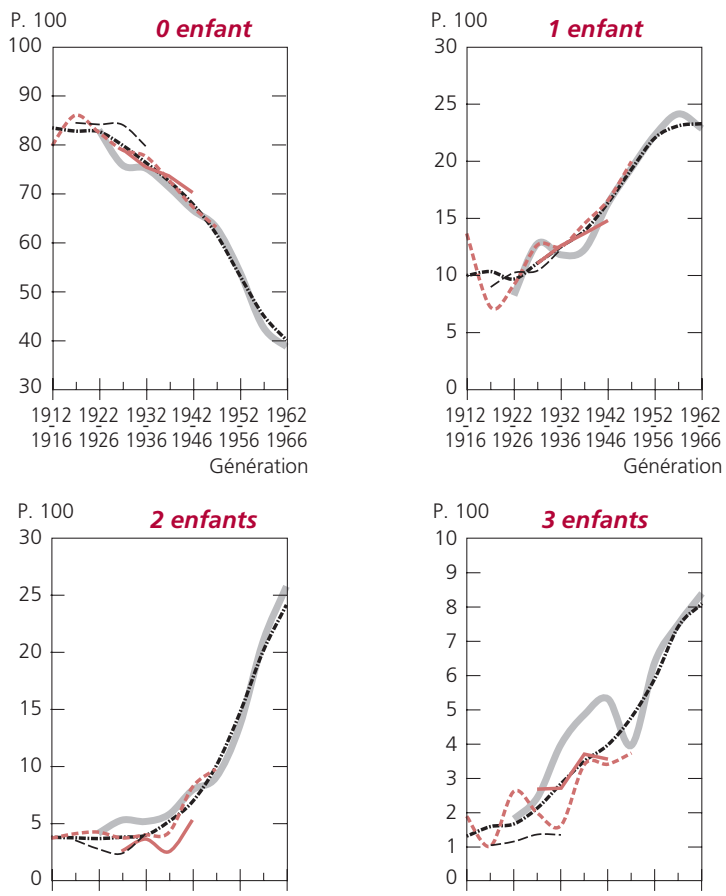

191219221932194219521962 $19 \overline{16} 192619 \overline{3} 619 \overline{4} 619561966$

191219221932194219521962 191619261936194619561966

Génération

Génération
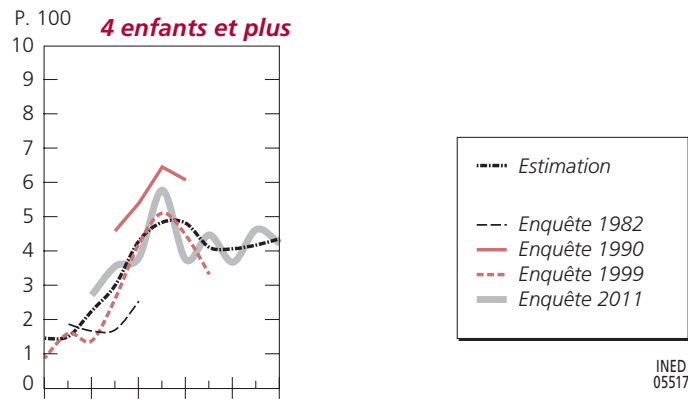

INED
05517

191219221932194219521962

191619261936194619561966

Génération

Source : Calculs de l'auteure à partir de Insee, Recensement de la population, 1931, 1946; Insee, ADISPCMH, enquête Famille, 1954, 1975, 1982, 1990; Insee, ADISP-CMH, Étude de I'histoire familiale, 1999; Insee, ADISP-CMH, Famille et logements, 2011. 
Figure A.3. Distribution des parités pour l'ensemble des femmes

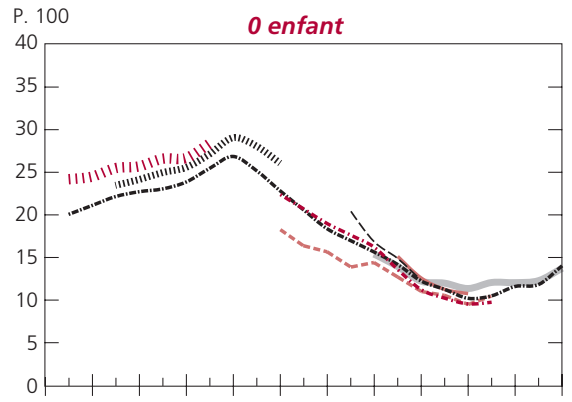

185618621872188218921902191219221932194219521962 et av. 18661876188618961906191619261936194619561966

Génération

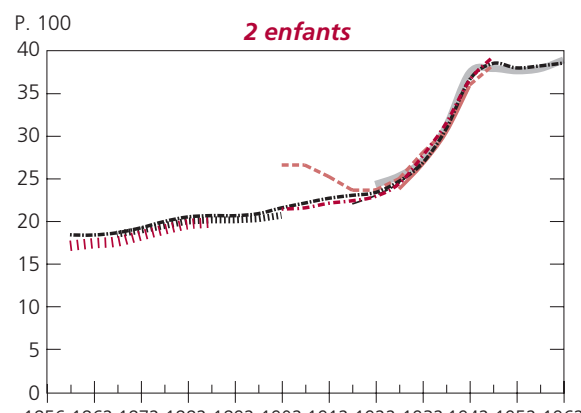

185618621872188218921902191219221932194219521962 et av. $18 \overline{6} 618 \overline{7} 18 \overline{8} 618961906191619261936194619561966$

Génération

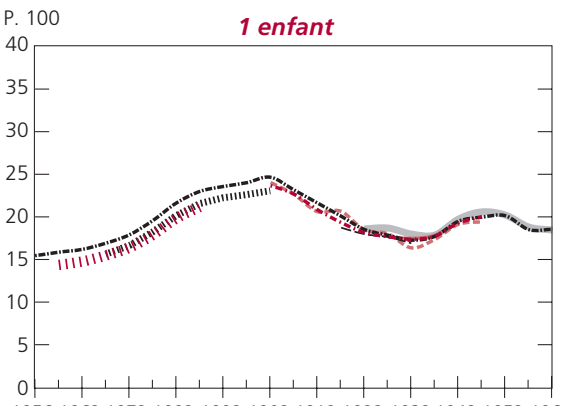

185618621872188218921902191219221932194219521962 et av. 18661876188618961906191619261936194619561966

Génération

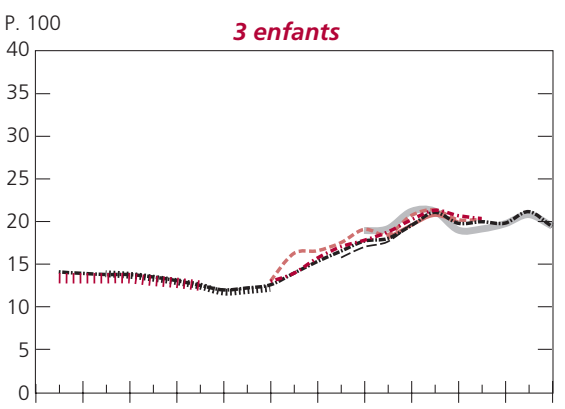

185618621872188218921902191219221932194219521962 et av. 18661876188618961906191619261936194619561966 Génération
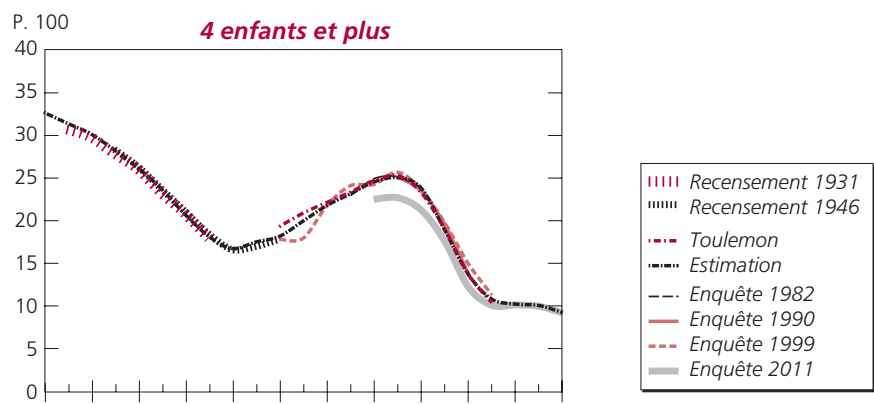

INED
05617

185618621872188218921902191219221932194219521962 et av. 18661876188618961906191619261936194619561966

Génération

Note : Les résultats des recensements de 1931 et de 1946 ne sont donnés qu'à titre indicatif puisqu'ils ont été calculés comme si les femmes célibataires n'avaient eu aucun enfant (ce qui explique les décalages bien plus élevés pour les 0 et les 1 enfant que pour les autres parités).

Source : Insee, Recensement de la population, 1931, 1946; Insee, ADISP-CMH, enquête Famille, 1954, 1975, 1982, 1990; Insee, ADISP-CMH, Étude de I'histoire familiale, 1999; Insee, ADISP-CMH, Famille et logements, 2011. 
Figure A.4. Descendance finale des générations selon différentes estimations

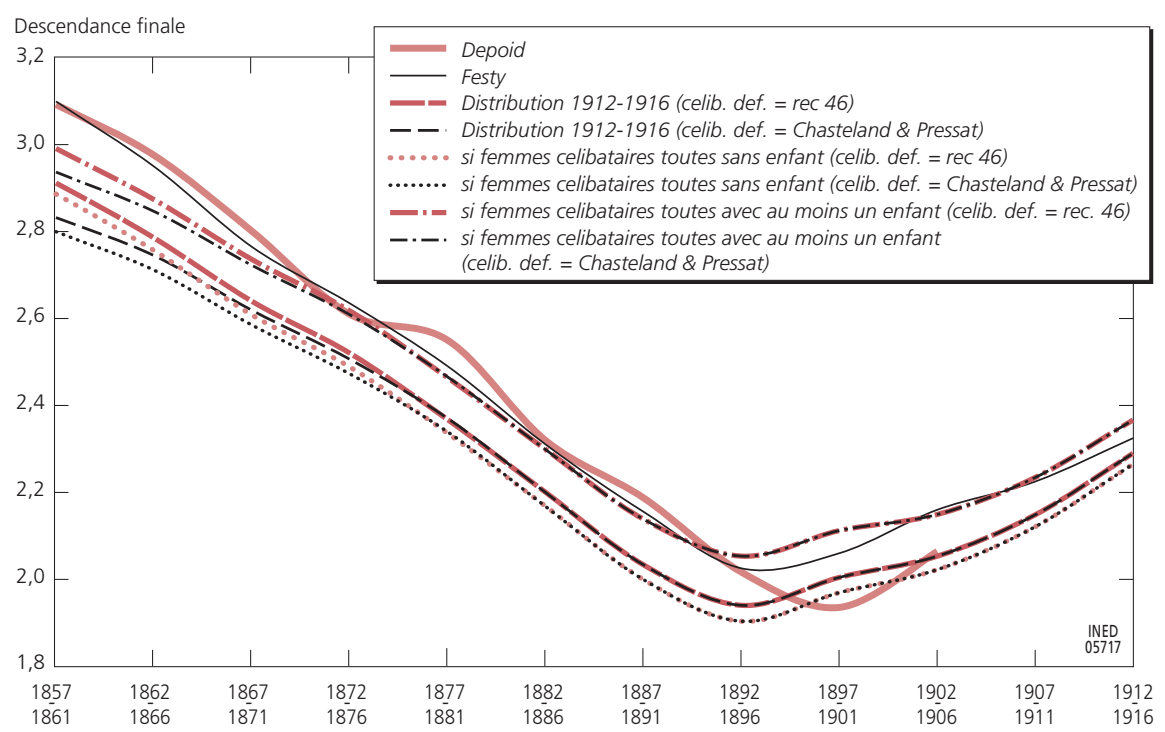

Générations quinquennales

Lecture : "Si les femmes célibataires avaient toutes au moins 1 enfant »: $90 \%$ avec 1 enfant, $5 \%$ avec 2 enfants, $3 \%$ avec 3 enfants et $2 \%$ avec 4 enfants ou plus.

Sources : Depoid (1941), Festy (1979), Chasteland et Pressat (1962). Estimations de la descendance finale des générations 1857-1916 selon diverses sources et selon que l'on utilise le niveau de célibat du recensement de 1946 ou celui estimé dans Chasteland et Pressat (1962). 
Figure A.5. Simulation de la descendance finale et des parités des femmes déjà mariées en l'absence de remontée de la proportion des familles nombreuses entre les générations 1897-1901 et 1942-1946

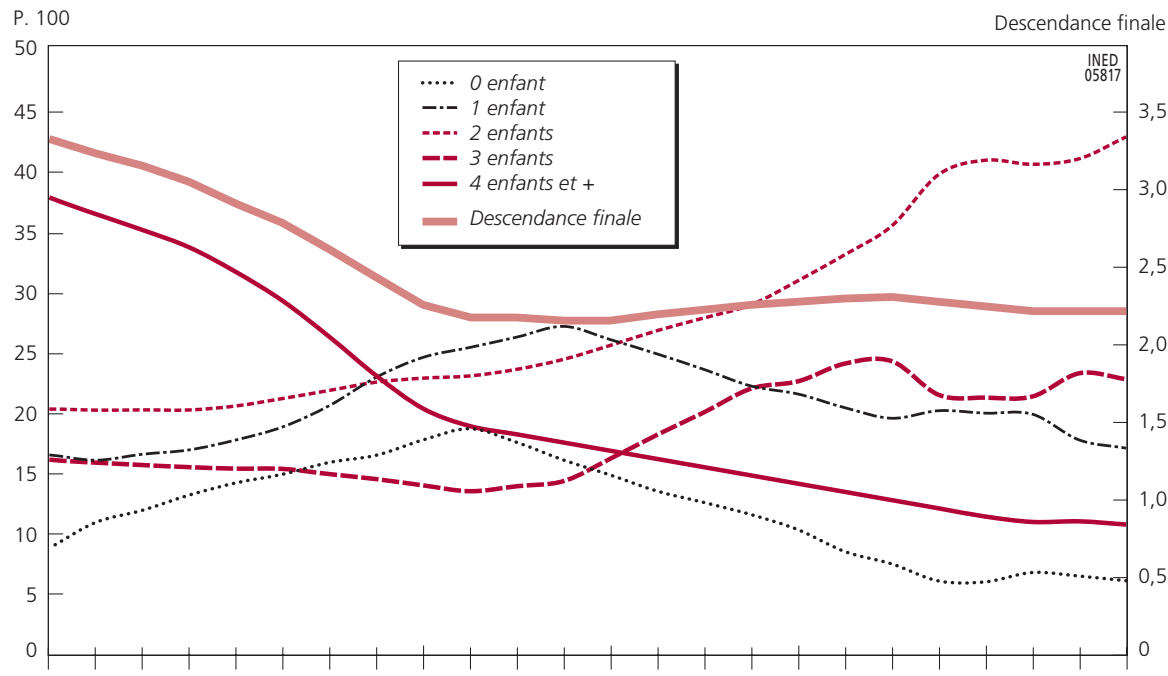

< 18521857186218671872187718821887189218971902190719121917192219271932193719421947195219571962

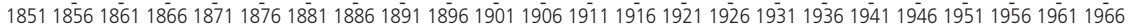

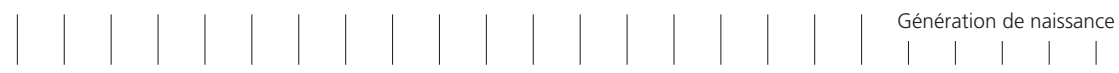

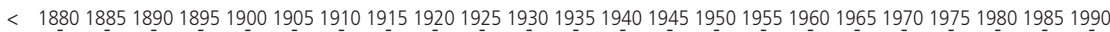
187918841889189418991904190919141919192419291934193919441949195419591964196919741979198419891994

+28 ans*

* L'âge moyen à la naissance est de 28 ans pour les générations les plus anciennes, et de 27 ans pour les plus récentes (Insee, estimations de population et statistiques de l'état civil). Pour plus de facilité, l'âge moyen de

28 ans a été ajouté aux générations de naissance pour donner une idée de la période de fécondité.

Note : Cette estimation fait baisser linéairement la proportion des familles nombreuses de 13,25\% pour les générations 1937-1941 à 11,89\% pour les générations 1947-1951, faisant disparaître complètement la remontée observée entre ces deux groupes de générations. La part restante est ventilée dans les autres parités en fonction de leur répartition effectivement observée.

Source : Calculs de l'auteure à partir de Insee, Recensement de la population, 1931, 1946; Insee, ADISPCMH, enquête Famille, 1954, 1975, 1982, 1990; Insee, ADISP-CMH, Étude de I'histoire familiale, 1999; Insee, ADISP-CMH, Famille et logements, 2011. 
Figure A.6. Probabilités d'agrandissement pour les femmes déjà mariées nées entre 1850 et 1966.

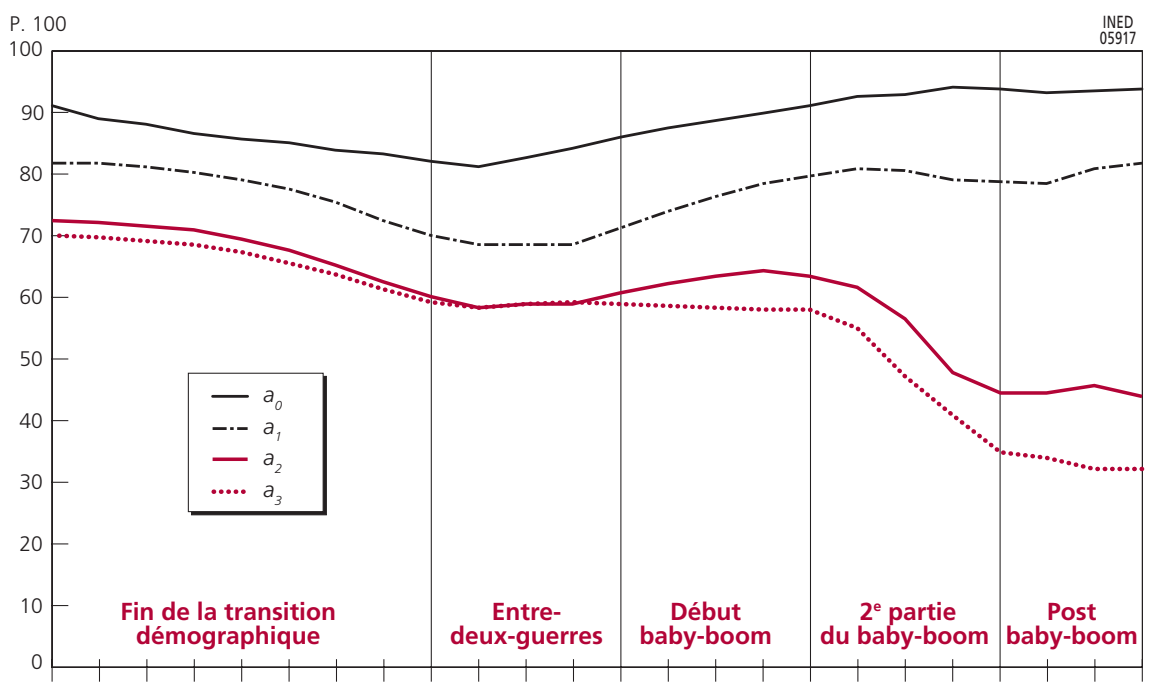

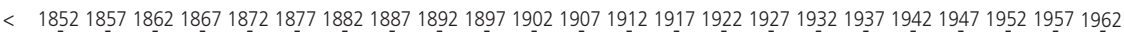

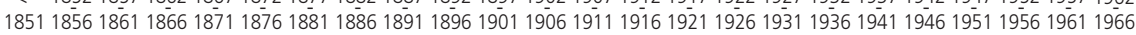<smiles>CCC</smiles>

< 18801885189018951900190519101915192019251930193519401945195019551960196519701975198019851990

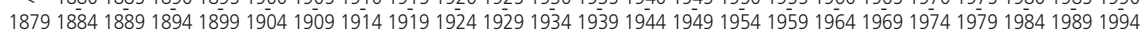

$$
+28 \text { ans* }
$$

* L'âge moyen à la naissance est de 28 ans pour les générations les plus anciennes, et de 27 ans pour les plus récentes (Insee, estimations de population et statistiques de l'état civil). Pour plus de facilité, l'âge moyen de

28 ans a été ajouté aux générations de naissance pour donner une idée de la période de fécondité.

Note : Les probabilités d'agrandissements pour les générations nées après 1962 sont données à titre indicatif (source : enquête Famille et logements, 2011).

Source : Calculs de l'auteure à partir de Insee, Recensement de la population, 1931, 1946; Insee, ADISPCMH, enquête Famille, 1954, 1975, 1982, 1990; Insee, ADISP-CMH, Étude de I'histoire familiale, 1999; Insee, ADISP-CMH, Famille et logements, 2011. 


\section{SOURCES STATISTIQUES}

Enquête Famille, 1954, Insee, ADISP-CMH.

Enquête Famille, 1975, Insee, ADISP-CMH.

Enquête Famille, 1982, Insee, ADISP-CMH.

Enquête Famille, 1990, Insee, ADISP-CMH.

Étude de l'histoire familiale, 1999, Insee, ADISP-CMH.

Famille et logements, 2011, Insee, ADISP-CMH.

Recensement de la population, 1931, Statistique générale de la France

Recensement de la population, 1946, Insee.

\section{RÉFÉRENCES}

ALTER Georges, 1992, "Theories of fertility decline: A non-specialist's guide to the current debate », in Gillis John. R., Tilly Louise A., Levine David (eds.), The European Experience of Declining Fertility, 1850-1970. The Quiet Revolution, Cambridge, Massachusetts, Blackwell, p. 13-27.

ANDERSON Michael, 1998, « Highly restricted fertility: Very small families in the British fertility decline », Population Studies, 52(2), p. 177-199.

ANDERSSON Gunnar, SOBOLEV Boris, 2013, « Small effects of selective migration and selective survival in retrospective studies of fertility ", Revue européenne de démographie, 29(3), p. 345-354.

ARIĖS Philippe, 1960, L'enfant et la vie familiale sous l'Ancien Régime, Paris, Plon, 316 p.

ARIÈS Philippe, 1980, « Two successive motivations for the declining birth rate in the West », Population and Development Review, 6(4), p. 645-650.

BECKER Gary, 1965, «A theory of the allocation of time », The Economic Journal, 75(299), p. 493-517.

Bonvalet Catherine, ClÉment Céline, OGG Jim, 2011, Réinventer la famille, l'histoire des baby-boomers, Paris, PUF, Le Lien social, 373 p.

BRÉE Sandra, 2011, "La fécondité à Paris et dans sa région au XIX $X^{e}$ siècle ", thèse de doctorat, Paris Sorbonne.

BRÉE Sandra, 2014, « Incidence de la fécondité illégitime sur la fécondité générale à Paris au XIX ${ }^{e}$ siècle », Espace, population, société, volume 1, 22 p.

BRÉE Sandra, 2017, Paris l'inféconde. La limitation des naissances en région parisienne au XIX $X^{e}$ siècle, Paris, Ined, Études et enquêtes historiques, 240 p.

BRÉE Sandra, EGGERICKX Thierry, SANDERSON Jean-Paul, 2017, « Low fertilities, childlessness and family changes in the first half of the $20^{\text {th }}$ century in France and Belgium », Revue Quetelet, 5(1), p. 7-31.

BréE Sandra, Bourguignon Mélanie, EGGERICKX Thierry, 2016a, « La fécondité en Europe occidentale durant l'entre-deux-guerres. Quels effets des crises sur les comportements démographiques? ", Annales de démographie historique, 132(2), p. 41-63. 
BRÉE Sandra, EgGERICKX Thierry, SANDERSOn Jean-Paul, Costa Rafael,2016b, « Comparaison des données rétrospectives de fécondité dans les recensements en Belgique et les enquêtes Famille en France », Population, 71(1), p. 85-120.

Brée Sandra, De LuCA-BARrusse Virginie, EGgerickx Thierry, Praz AnneFrançoise, 2016c, « Avoir des enfants? Les transitions de fécondité depuis la fin du XIX siècle ", Annales de démographie historique, 132(2), p. 7-16.

Breschi Marco, Esposito Massimo, Fornasin Alessio, Manfredini Matteo, 2016, « Reproductive change in transitional Italy. More insights from the italian fertility survey of $1961 »$, Annales de démographie historique, 132, p. 111-137.

BRETON Didier, PRIOUX France, 2009, « The one-child family: France in the European context », Demographic Research, 20(27), p. 657-692.

CALDWELL John C., 1976, « Toward a restatement of demographic transition theory ", Population and Development Review, 2(3-4), p. 321-366.

Caldwell John C., 2001, «The globalization of fertility behavior », Population and Development Review, 27, Supplement: Global Fertility Transition, p. 93-115.

CALOT Gérard, SARDON Jean-Paul, 1998, « La vraie histoire du baby-boom », Sociétal, 16, p. 41-44.

Chasteland Jean-Claude, PRessat Rolland, 1962, « La nuptialité des générations françaises depuis un siècle », Population, 17(2), p. 215-240.

CHESNAIS Jean-Claude, 2006, « La politique de population en France, 1896-2003 », in Caselli Graziella, Vallin Jacques, Wunsch Guillaume (dir.), Démographie: Analyse et synthèse, volume VII, Paris, Ined, Les Manuels, p. 797-832.

COLEMAN David, 2004, "Why we don't have to believe without doubting in the "Second Demographic Transition" - some agnostic comments », Vienna Yearbook of Population Research, 2(1), p. 11-24.

COVA Anne, 1997, Maternité et droits des femmes en France, XIX $X^{e}$-XX $X^{e}$ siècles, Paris, Anthropos, Historiques, $435 \mathrm{p}$.

DAGUET Fabienne, 2002, «Un siècle de fécondité française. Caractéristiques et évolution de la fécondité de 1901 à 1999 », Paris, Insee résultats, société nº 8, 305 p.

DE LUCA-BARRUSSE Virginie, 2008, Les familles nombreuses, Rennes, PUR, 342 p.

DEPOID Pierre, 1941, « Reproduction nette en Europe depuis les origines des statistiques de l'état civil », Paris, Statistique générale de la France, Études démographiques $\mathrm{n}^{\circ} 1,42 \mathrm{p}$.

DESPLANQUES Guy, 1988, « La fécondité de 1939 à 1964 : le baby-boom », in Dupâquier J. (dir.), Histoire de la population française, tome IV. De 1914 à nos jours, Paris, PUF, p. 297-309.

DOLIGER Cédric, 2008, « La fécondité et ses déterminants économiques : Becker vs Easterlin », Revue économique, 59(5), p. 955-971.

DORBRITZ Jürgen, SCHWARTZ Karl, 1996, « Kinderlosigkeit in Deutschland-ein Massenphänomen? Analysen zu Erscheinungsformen und Ursachen » [L'infécondité en Allemagne, un phénomène de masse? Analyse des manifestations et des causes], Zeitschrift für Bevölkerungswissenschaft, 21, p. 231-261.

DUVOISIN Aline, BURGNARD Sylvie, ORIS Michel, 2016, « Childless people during the baby boom in Switzerland », Annales de démographie historique, 132(2), p. 193-221.

EGGERICKX Thierry, BEGEOT François, 1993, « Les recensements en Europe dans les années 1990. De la diversité des pratiques nationales à la comparabilité internationale des résultats », Population, 48(6), p. 1705-1732.

EGGERICKX Thierry, BrÉE Sandra, BOURGuignON Mélanie, 2016, « Transition de la fécondité et évolution économique du $18^{\mathrm{e}}$ au $21^{\mathrm{e}}$ siècle. L'exemple de la Wallonie ", Revue belge d'histoire contemporaine, 46(3-4), p. 46-74. 
FAUVE-ChAmoux Antoinette, BRUnet Guy, 2014, « L'enfant illégitime et ses parents, tendances européennes et coloniales au XIX ${ }^{\mathrm{e}}$ siècle, au sein des modèles séculaires d'illégitimité », Annales de démographie historique, 127(1), p. 7-43.

FESTY Patrick, 1979, La fécondité des pays occidentaux de 1870 à 1970, Paris, Ined, Cahier $\mathrm{n}^{\circ} 85,400 \mathrm{p}$.

FREJKA Tomaš, 2008, « Overview Chapter 2: Parity distribution and completed family size in Europe: Incipient decline of the two-child family model? », Demographic Research, 19, p. 47-72.

Gauvreau Danielle, LAPlante Benoît, 2016, « La fécondité au Canada durant le baby-boom. Divergence et convergence des comportements », Annales de démographie historique, 132(2), p. 65-110.

GuiberT-LANTOINE Catherine, LeRIdOn Henri, Toulemon Laurent, VilleneuveGOKALP Catherine, 1994, « La cohabitation adulte », Population et sociétés, n² 293, 4 p.

Henry Louis, 1953, "Vue sur la statistique des familles », Population, 8(3), p. 473-490.

HÉRAN François, 2005, Préface « Du public, du privé et de l'intime dans l'enquête Famille 1999. Témoignage d'un concepteur sur une enquête qui revient de loin », in Lefèvre C., Filhon A. (dir.), Histoires de familles, histoires familiales. Les résultats de l'enquête Famille de 1999, Paris, Ined, Cahiers, nº 156, p. XI-XXXVIII.

INED, 1966, « Rapport de l'Institut national d'études démographiques à Monsieur le Ministre des Affaires sociales sur la régulation des naissances en France », Population, 21(4), p. 645-690.

INED, 1976, « Rapport sur la situation démographique de la France en 1974 », Population, 31(1), p. 15-62.

INED, 1991, «Vingtième rapport sur la situation démographique de la France », Population, 46(5), p. 1081-1159.

KNIBIEHLER Yvonne, 1997, La Révolution maternelle depuis 1945, Paris, Perrin, 370 p.

LERIDON Henri, 1987, La seconde révolution contraceptive. La régulation des naissances en France de 1950 à 1985, Paris, Ined, Cahiers, n 117, 380 p.

Lesthaeghe Ron, VAn DE KAA Dick, 1986, « Twee Demografische Transities? [Two Demographic transitions?], in Van de Kaa D., Lesthaeghe R. (eds.), Bevolking: Groei en Krimp [Population: Growth and Decline], Deventer, Van Loghum Slaterus. p. 9-24.

LESTHAEGHE Ron, 1995, « La deuxième transition démographique dans les pays occidentaux : une interprétation », in Tabutin D., Eggerickx T., Gourbin C. (dir.), Transitions démographiques et sociétés, Chaire Quetelet 1992, Institut de démographie, Louvain-la-Neuve, Académia/L'Harmattan, p. 133-189.

MCLAREN Angus, 1996, Histoire de la contraception, Paris, Noësis, 413 p.

MASSON Luc, 2013, « Avez-vous eu des enfants? Si oui, combien? ", France, portrait social, Paris, Insee références, p. 9-109.

MAZuY Magali, Toulemon Laurent, 2013, « Fécondité des hommes et des femmes selon le niveau d'études d'après l'enquête EFL », Ined, Réunion du groupe d'exploitation de l'enquête Famille et logements (EFL).

MAZUY Magali, BARBIERI Magali, D'ALBIS Hippolyte, 2013, « L'évolution démographique récente en France : la fécondité est stable », Population, 68(3), p. 385-432.

MORGAN Phillip S., 1991, « Late nineteenth-and early twentieth-century childlessness », American Journal of Sociology, 97(3), p. 779-807.

MuÑOZ-PEREZ Francisco, PRIOUX France, 1999, « Naître hors mariage », Population et Sociétés, $\mathrm{n}^{\circ} 342,4 \mathrm{p}$. 
NEELS Karel, 2010, « Period and cohort effects in Belgian nuptiality and fertility: 1960-1990 », in Eggerickx T., Sanderson J-P. (dir.), Histoire de la population de la Belgique et de ses territoires, Actes de la Chaire Quetelet 2005, Louvain-la-Neuve, Presses universitaires de Louvain, p. 463-488.

NiZARD Alfred, MAKSUD Monique, 1977, «Enfants trouvés, reconnus, légitimés. Les statistiques de la filiation en France, aux XIX ${ }^{e}$ et $\mathrm{XX}^{\mathrm{e}}$ siècles », Population, 32(6), p. 1159-1220.

NOMES Eli, VAN BAVEL Jan, 2016, « Marital fertility and educational assortative mating before, during, and after the baby boom in Belgium », Annales de démographie historique, 132(2), p. 139-163.

PRAZ Anne-Françoise, 2005, De l'enfant utile à l'enfant précieux. Filles et garçons dans les cantons de Vaud et Fribourg (1860-1930), Lausanne, Antipodes, 652 p.

PRIOUX France, 2002, "L'évolution démographique récente en France », Population 57(4-5), p. 691-732.

RAUlT Wilfried, RÉGNIER-LOILIER Arnaud, 2015, « La première vie en couple : évolutions récentes ", Population et sociétés, n 521, 4 p.

Rebreyend Anne-Claire, 2003, «Sexualités vécues. France 1920-1970 », Clio, Mixité et coéducation, $\mathrm{n}^{\circ} 18$, p. 209-222.

ROUSSEL Louis, 1978, « La cohabitation juvénile en France », Population, 33(1), p. 15-42.

ROWLAND Donald T., 2007, « Historical trends in childlessness ", Journal of Family Issues, 28(10), p. 1311-1337.

RUSTERLHOZ Caroline, 2015, « Reproductive behavior and contraceptive practices in comparative perspective, Switzerland (1955-1970) », The History of the Family, 20(1), p. 41-68.

RUSTERHOlz Caroline, 2017, Deux enfants c'est déjà pas mal. Famille et fécondité en Suisse (1955-1970), Lausanne, Antipodes, Histoire, 470 p.

SARDON Jean-Paul, 1990, La fécondité des cohortes dans les États membres du Conseil de l'Europe, Conseil de l'Europe, Études démographiques, n 21, 205 p.

SoHn Anne-Marie, 1996, Du premier baiser à l'alcôve, la sexualité des Français au quotidien (1850-1950), Paris, Aubier, Collection historique, 310 p.

TOULEMON Laurent, 1995, « Très peu de couples restent volontairement sans enfant », Population, 50(4-5), p. 1079-1109.

TOUlEmON Laurent, 2001, «Combien d'enfants, combien de frères et sœurs depuis cent ans? ", Population et sociétés, $\mathrm{n}^{\circ} 374,4 \mathrm{p}$.

TOUlemon Laurent, PAilhé Ariane, ROSSIER Clémentine, 2008, « France: High and stable fertility », Demographic Research, 19(16), p. 503-556.

VAN BAVEL Jan, 2014, "The mid-twentieth century Baby Boom and the changing educational gradient in Belgian cohort fertility », Demographic Research, 30(33), p. 925-962.

VAN BAVEL Jan, KOK Jan, 2010, « Pioneers of the modern life style? Childless couples in early twentieth century Netherlands », Social Science History, 34(1), p. 47-72.

VAN BAVEL Jan, REHER David, 2013, « The Baby-Boom and its causes: What we know and what we need to know », Population and Development Review, 39(2), p. 257-288.

VAN DE KAA Dick J., 1987, « Europe's second demographic transition », Population Bulletin, 42(1), p. 1-59.

VinCENT Paul, 1946, «L'utilisation des statistiques des familles », Population, 1(1), p. 143-148.

Westoff Charles F., RYder Norman B., 1977, The Contraceptive Revolution, Princeton, Princeton University Press, 398 p. 


\section{Sandra BRÉE • Évolution DE LA TAILLE DES FAMILLES AU FIL DES GÉNÉRATIONS EN FRANCE} (1850-1966)

À travers l'analyse longitudinale des parités et des probabilités d'agrandissement, cet article retrace l'évolution de la fécondité des générations féminines en France nées entre 1850 et 1966 . Après avoir présenté les sources et données disponibles (recensements de population et enquêtes Famille), et les méthodes utilisées pour l'analyse rétrospective de la fécondité des générations, l'auteure propose des estimations de l'évolution de la taille des familles sur plus d'un siècle. L'étude de l'évolution conjointe de la taille des familles et de la descendance finale améliore la compréhension des tendances de la fécondité, et plus particulièrement le poids de l'évolution de chaque taille de famille dans l'évolution générale de la fécondité. Elle nuance ainsi les conclusions que l'on pourrait tirer de la lecture des simples moyennes (descendances finales) en montrant qu'à descendance finale égale, la distribution des parités peut être très variable, et encourage à toujours prendre en compte la composition des familles dans l'analyse de la fécondité. Cette recherche souligne également l'intérêt de la différenciation de la fécondité de l'ensemble des femmes, des femmes ayant été mariées et des femmes célibataires, notamment dans l'analyse de l'infécondité et des basses fécondités (1 enfant).

\section{Sandra Brée • Changes in Family Size OVer the Generations In France (1850-1966)}

Through a longitudinal analysis of parities and parity progression ratios, this article charts the fertility of the cohorts of women born in France between 1850 and 1966. After reviewing the available sources and data (population censuses and family surveys), and the methods used for retrospective cohort analysis of fertility, the author proposes estimates of changing family sizes over more than a century. The simultaneous study of trends in family size and completed fertility enhances our understanding of fertility trends, and more specifically the impact of changes in each family size on overall fertility patterns. This refines the conclusions that can be drawn from an interpretation of averages (completed fertility) by showing that, for the same completed fertility, the parity distribution can be highly variable, and demonstrates that family size should always be taken into account when analysing fertility. This research also highlights the value of differentiating between the fertility of all women, of ever-married women and of never-married women, particularly when analysing childlessness and low fertility (one child).

\section{Sandra BRÉE • EvoluCión DEL TAMAÑo de LAS FAMILIAS A LO LARGO DE LAS GENERA- CIONES EN FRANCIA (1850-1966)}

Conjugando el análisis longitudinal de las paridades y de las probabilidades de crecimiento de las familias, este artículo sigue la evolución de la fecundidad de las generaciones femeninas en Francia, nacidas entre 1850 y 1966. Después de presentar las fuentes y los datos disponibles (censos de la población y encuestas Famille), así como los métodos utilizados para el análisis retrospectivo de la fecundidad, la autora propone estimaciones de la evolución del tamaño de las familias durante más de un siglo. El estudio conjunta del tamaño de las familias y de la descendencia final mejora la comprensión de las tendencias de la fecundidad, y en especial el papel que ha jugado cada tamaño de familia en la evolución general de la fecundidad. Se matizan así las conclusiones que se podrían sacar de la simple lectura de las descendencias finales, y se muestra que para una misma descendencia final la distribución según el tamaño de las familias puede ser muy variable. Lo cual incita a tener siempre en cuenta la composición de las familias en el análisis de la fecundidad. En el artículo se subraya igualmente el interés de distinguir la fecundidad del conjunto de las mujeres, la de las mujeres casadas y la de las mujeres solteras, sobre todo en el análisis de la infecundidad y de la baja fecundidad (un hijo).

Mots-clés : fécondité, générations, France, analyse longitudinale, analyse rétrospective, démographie historique

Keywords: fertility, cohorts, France, longitudinal analysis, retrospective analysis, historical demography 